\title{
Intracellular Correlates of Stimulus-Specific Adaptation
}

\author{
Itai Hershenhoren, ${ }^{1,2}$ Nevo Taaseh, ${ }^{1,2}$ Flora M. Antunes, ${ }^{1,3}$ and Israel Nelken ${ }^{1,2,3}$ \\ ${ }^{1}$ Department of Neurobiology, Institute of Life Sciences, ${ }^{2}$ The Interdisciplinary Center for Neural Computation, and ${ }^{3}$ The Edmond and Lily Safra Center for \\ Brain Sciences, The Hebrew University of Jerusalem, Jerusalem 91904, Israel
}

\begin{abstract}
Stimulus-specific adaptation (SSA) is the reduction in response to a common stimulus that does not generalize, or only partially generalizes, to rare stimuli. SSA is strong and widespread in primary auditory cortex (A1) of rats, but is weak or absent in the main input station to A1, the ventral division of the medial geniculate body. To study SSA in A1, we recorded neural activity in A1 intracellularly using sharp electrodes. We studied the responses to tone pips of the same frequency in different contexts: as Standard and Deviants in Oddball sequences; in equiprobable sequences; in sequences consisting of rare tone presentations; and in sequences composed of many different frequencies, each of which was rare. SSA was found both in subthreshold membrane potential fluctuations and in spiking responses of A1 neurons. SSA for changes in frequency was large at a frequency difference of $44 \%$ between Standard and Deviant, and clearly present with tones separated by as little as $4 \%$, near the behavioral frequency difference limen in rats. When using equivalent measures, SSA in spiking responses was generally larger than the SSA at the level of the membrane potential. This effect can be traced to the nonlinearity of the transformation between membrane potential to spikes. Using the responses to the same tone in different contexts made it possible to demonstrate that cortical SSA could not be fully explained by adaptation in narrow frequency channels, even at the level of the membrane potential. We conclude that local processing significantly contributes to the generation of cortical SSA.
\end{abstract}

\section{Introduction}

In the human auditory system, a "Deviant" sound presented within a sequence of a repeating "Standard" tone (an "Oddball sequence") elicits specific electrical responses that occur first in the midlatency potentials (Grimm and Escera, 2012), which are associated with the earliest activation of auditory cortex, followed by the well studied mismatch negativity (Naatanen et al., 1978). Auditory neurons in animal models show a similar, although not identical (Farley et al., 2010), behavior. Ulanovsky et al. (2003) have shown that, when presented with Oddball sequences, single units in the cat primary auditory cortex (A1) responded more strongly to a tone when Deviant than when Standard. They adopted the term stimulus-specific adaptation (SSA; Movshon and Lennie, 1979) for this phenomenon. While SSA is absent in the cochlear nucleus (Ayala et al., 2012), it is present in the mammalian inferior colliculus (Pérez-González et al., 2005; Malmierca et al., 2009; Zhao et al., 2011; Thomas et al., 2012), auditory thalamus (Anderson et al., 2009; Antunes et al., 2010; Antunes and Malmierca, 2011), and A1 (Ulanovsky et al., 2003, 2004; von der Behrens et al., 2009; Taaseh et al., 2011; Fishman and Steinschneider, 2012; Yaron et al., 2012), as well as in the midbrain and

Received May 22, 2013; revised Jan. 1, 2014; accepted Jan. 19, 2014.

Author contributions: I.H., N.T., and I.N. designed research; I.H., N.T., F.M.A., and I.N. performed research; I.H.,

N.T., and I.N. analyzed data; I.H. and I.N. wrote the paper.

The authors declare no competing financial interests.

This research was supported by grants from the Israeli Science Foundation, the German-Israeli Foundation, the US-Israel Binational Science Foundation, Israel Ministry of Health under the framework of ERA-NET-NEURON (consortium PANS), and the Gatsby (haritable Foundation.

Correspondence should be addressed to Israel Nelken, Department of Neurobiology, The Alexander Silberman Institute of Life Sciences, Edmond Safra Campus, Givat Ram, Jerusalem 91904, Israel. E-mail: israel@cc.huji.ac.il.

DOI:10.1523/JNEUROSCI.2166-13.2014

Copyright $\odot 2014$ the authors $\quad 0270-6474 / 14 / 343303-17 \$ 15.00 / 0$ forebrain of birds (Reches et al., 2010; Netser et al., 2011; Gutfreund, 2012). Subcortically, SSA has been found mostly in the nonlemniscal pathway. In contrast, the main thalamic input to A1 [the ventral division of the medial geniculate body (vMGB)] exhibits weak or no SSA (Anderson et al., 2009; Antunes et al., 2010; Bäuerle et al., 2011). Thus, A1 is the first lemniscal station where SSA is widespread and strong. Indeed, we have recently demonstrated SSA at the level of membrane potential $\left(V_{\mathrm{m}}\right)$ in A1 neurons (Yaron et al., 2012).

A previous study of extracellular responses in rat A1 (Taaseh et al., 2011) has shown that the habituation to the Standard could affect adjacent frequencies, although to a lesser degree. Taaseh et al. (2011) studied a model that postulated the existence of "adaptation channels" (estimated to have a width of approximately one-third of an octave) to account for SSA (Mill et al., 2011). Indeed, thalamocortical synapses show synaptic depression, which may instantiate the adaptation channels. The model has been tested by using additional tone sequences, including a "rare" condition (where a single tone frequency was presented, but very rarely) as well as multitone sequences. The model consistently underpredicted responses to Deviants in Oddball sequences. Taaseh et al. (2011) used this finding to argue for the existence of true deviance sensitivity in auditory cortex.

In this study, we used intracellular recordings to investigate thoroughly the SSAs of single neurons in rat auditory cortex. We show SSA already at the level of the sensory EPSP, which reflects the summed sound-driven inputs to the neuron, and its enhancement in the spiking activity. Finally, we demonstrate that adaptation in narrow frequency channels cannot account for the full magnitude of cortical SSA even at the level of the membrane 
potential, suggesting the presence of true deviance sensitivity in A1 neurons.

\section{Materials and Methods}

Preparation. Sixty adult female Sabra rats weighing 220-240 g were used for this study (Harlan Laboratories). The joint ethics committee of the Hebrew University and Hadassah Medical Center approved the study protocol for animal welfare. The Hebrew University is an Association for Assessment and Accreditation of Laboratory Animal Care Internationalaccredited institute.

Surgical procedures have been described in the studies by Taaseh et al. (2011) and Yaron et al. (2012). In short, animals were initially anesthetized with ketamine and medetomidine by intramuscular injection. Following a tracheotomy, the animals were ventilated through a tracheal cannula with a mixture of oxygen and halothane $(0.5-1.5 \%$, as needed) throughout the experiment. Using an inhaled anesthesia agent allowed the maintenance of a constant anesthesia level throughout the experimental procedure. Halothane anesthesia results in responses that are closer to awake responses than those recorded under other anesthetic agents (Moshitch et al., 2006). Temperature, respiratory $\mathrm{CO}_{2}$, and respiration quality were continuously monitored. The rats were anesthetized to a level in which they could be respirated, although they were not paralyzed. Stability of anesthesia was judged by the lack of withdrawal reflexes and by the stable waveform of the respiratory $\mathrm{CO}_{2}$.

The skull above left auditory cortex was exposed by removal of the skin, the temporal muscle, and connective tissues. A small craniotomy $(0.5-1 \mathrm{~mm})$ was performed over part of the estimated location of the auditory cortex. To reduce brain pulsation for more stable recordings, the dura covering the cisterna magna was exposed and was carefully perforated using a needle (30 ga). Sharp electrodes were prepared from a filamented borosilicate tube $(1.5 \mathrm{~mm}$ outer diameter, $0.86 \mathrm{~mm}$ inner diameter; Sutter Instruments) by a single-stage vertical puller (PE-2, Narishige) and were filled with a $1 \mathrm{~m}$ potassium acetate solution. A small durotomy was then performed, and the electrode was advanced into the cortex in small steps using a microdrive (MP-225, Sutter Instrument Company). Electrode resistance was in the range of 45-95 M $\Omega$. The bridge was balanced, and capacitance compensation was used in all experiments. Agarose gel (3-4\% agarose type III-A, Sigma), in saline solution, was used to decrease brain pulsation and to keep the surface of the brain moist. The signal was amplified $10 \times$ (NeuroData IR283, Cygnus Technologies), sampled at $12.207 \mathrm{kHz}$ (RP2.1, TDT, Tucker-Davis Technologies), displayed on-line and stored for off-line analysis. A blind search for neurons was conducted $400-1000 \mu \mathrm{m}$ below the surface to record neurons at the estimated depth of layer IV $(500-750 \mu \mathrm{m})$.

Auditory stimulation. Experiments were conducted in a sound-proof chamber (IAC). Sounds were generated on-line using a custom Matlab (MathWorks) application and transduced to voltage signals by a sound card (HDSP9632, RME) at a sampling rate of $192 \mathrm{kHz}$. Sounds were attenuated (PA5, Tucker-Davis Technologies) and played through a sealed speaker (EC1, Tucker-Davis Technologies) directly to the ear canal of the rat's right ear. In some animals, sound calibration was conducted using a custom-made adaptor for a miniature microphone (model EK-3133-000, Knowles), which was precalibrated relative to a $\mathrm{B} \& \mathrm{~K}$ one-quarter inch microphone. Attenuation level of $0 \mathrm{~dB}$ corresponded to $\sim 100 \mathrm{~dB}$ SPL for pure tones and noise stimuli were synthesized at a spectrum level of $-50 \mathrm{~dB} / \sqrt{ } \mathrm{Hz}$ relative to pure tones at the same attenuation level. Calibration was stable across all animals.

Experimental procedure. We report here the responses of 79 neurons that had stable recordings, lasting between 30 and 780 min. Auditory sensitivity was verified with 280 bursts of broadband noise (duration 200 $\mathrm{ms}, 10 \mathrm{~ms}$ linear onset and offset ramps, interstimulus interval (ISI; onset to onset) of $500 \mathrm{~ms}$ at seven attenuation levels, between 0 and $-60 \mathrm{~dB}$ with $10 \mathrm{~dB}$ steps (sound levels are expressed as attenuation levels). Each level was presented 40 times pseudorandomly. The main data were collected if the noise threshold was below $-30 \mathrm{~dB}$ and membrane potential fluctuations or spikes showed a clear dependence on sound level. All neurons presented here passed this test. To determine the frequency selectivity of a neuron, we used quasi-random frequency sequences of 370 tone bursts ( $50 \mathrm{~ms}, 5 \mathrm{~ms}$ onset/offset linear ramps; ISI of $500 \mathrm{~ms}$ ) at
37 frequencies (1-64 kHz, 6 tones/octave) at several attenuation levels, starting from approximately $-20 \mathrm{~dB}$ and then decreasing by $10 \mathrm{~dB}$ steps until the threshold of the neuron was found (threshold range, -20 to $-80 \mathrm{~dB}$, corresponding to $20-80 \mathrm{~dB} \mathrm{SPL}$ ). This procedure did not produce a significant amount of adaptation because the tones were rather widely spaced, and successive tones rarely occurred close to each other; furthermore, periods of silence of 30-60 s separated sequences at different sound levels. These data were used only to select the main frequencies and sound levels for the main experiment, and the responses to these tests are not reported here. Using these responses, the best frequency $(\mathrm{BF})$ was determined as the frequency that gave rise to the most consistent responses at all sound levels. Two frequencies evoking large responses were selected for further study on either side of the BF, and were denoted $f_{1}$ and $f_{2}$. The two frequencies were set to be $\mathrm{BF} /(1+D)$ and $\mathrm{BF} \times(1+D)$, were $D$ was $0.02,0.05,0.1$, and 0.2 . We defined the frequency separation between the two tones as $\Delta f=\left(f_{2}-f_{1}\right) / f_{1}$, which was $4 \%, 10 \%, 21 \%$, and $44 \%$. The last three are the same intervals used by Taaseh et al. (2011).

We used seven types of sequences at each $\Delta f$. In all tone sequences, tone duration was $30 \mathrm{~ms}$ ( $5 \mathrm{~ms}$ rise/fall time), presented most commonly with an ISI of $300 \mathrm{~ms}$ (we used ISIs of 700 and $1200 \mathrm{~ms}$ only for testing the dependence of SSA on ISI; see Fig. 5). Each sequence contained 500 trials. In the Oddball sequences, one tone was frequent (Standard) and was presented 475 times (95\%), and the other was rare (Deviant) and appeared 25 times (5\%) pseudorandomly. There were two Oddball sequences: in Deviant $f_{2}, f_{2}$ was rare (Deviant) and $f_{1}$ was common (Standard; Fig. $1 A$, schematic representation), and in Deviant $f_{1}, f_{1}$ was the Deviant and $f_{2}$ was the Standard (Fig. $1 B$ ). In the Equal sequence, each of the two frequencies occurred in $50 \%$ of the trials (Fig. 1C).

Two multitone sequences were used. In both sequences, $f_{1}$ and $f_{2}$ appeared with a probability of $5 \%$. However, the other $95 \%$ of sound presentations were distributed among many different frequencies, avoiding the regularity set by the Standard in the Oddball sequences. The first sequence included 20 tones distributed equally on a logarithmic scale over an interval of $\sim 2 \Delta f$. The frequencies included $f_{1}$ and $f_{2}$ (as the 6 th and 15th tones in increasing order). Each frequency was presented 25 times (5\%) randomly. This control block was termed "Diverse-narrow" (Fig. $1 D$ ). The other multitone sequence presented $f_{1}$ and $f_{2}$ together with 10 other tones (total of 12 tones). The frequency interval between each pair of adjacent frequencies was $\Delta f$. Tones of frequencies $f_{1}$ and $f_{2}$ were presented 25 times each (5\%), similar to the Deviants in the Oddball paradigm. The other 10 frequencies were presented in $9 \%$ of the tone presentations ( 45 times). The use of 12 tones rather than 20 in this block was due to the very wide frequency range over which the tones were distributed when $\Delta f=44 \%$ (for more details, see Taaseh et al. (2011)). This sequence was termed "Diverse-broad" (Fig. 1E). The Diverse-broad sequence is comparable to the "Deviant among many Standards," or control, condition used in some mismatch negativity studies (Jacobsen et al., 2003).

Finally, two sequences attempted to measure the unadapted responses to the two frequencies. In these sequences, tones of one frequency occurred in 25 of the trials (5\%) randomly, while the other 475 trials were silent. A separate sequence was used for each of the two frequencies. This condition was termed "Deviant-alone" (Fig. $1 F$ ).

The responses to each frequency were therefore tested in the following six different conditions: Standard, Deviant, Equal, Diverse-narrow, Diverse-broad, and Deviant-alone. We call the responses to one frequency in these six conditions a "set" of responses. Whenever possible, responses were recorded at multiple $\Delta f$ values, although $\Delta f=44 \%$ was used most commonly.

Data analysis. All data analyses were performed with Matlab. Spikes, when present, were detected and then clipped. To detect and clip the spikes, the raw signal from neurons that had spiking activity was first high-pass filtered with a corner frequency of $30 \mathrm{~Hz}$. Spikes were detected at the maximum point using a dynamic threshold that was 60 times the median of the absolute deviations from the median of the membrane potential. The signal was inspected to ensure the quality of spikes detection. The time of spike initiation was determined as the time of maximum acceleration of its rising phase, and its end point was determined as the time when the derivative of the membrane potential was closest to 
A

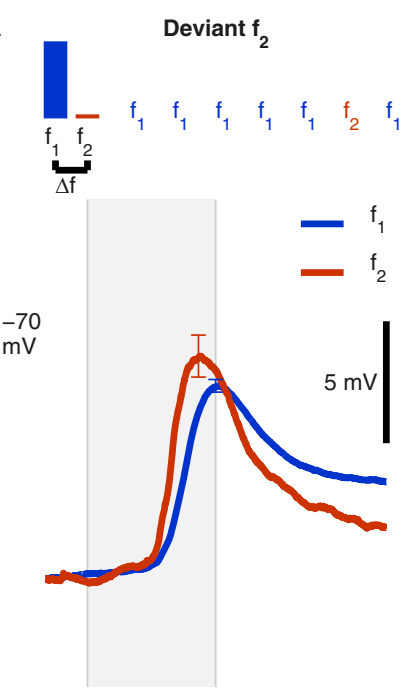

D

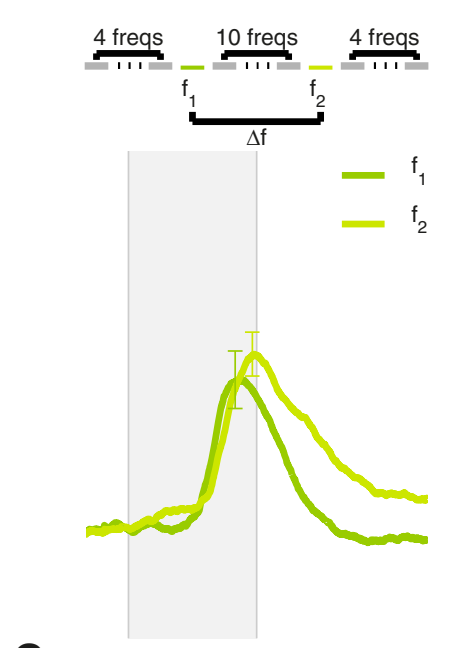

G

$$
f_{1} \text { all conditions }
$$

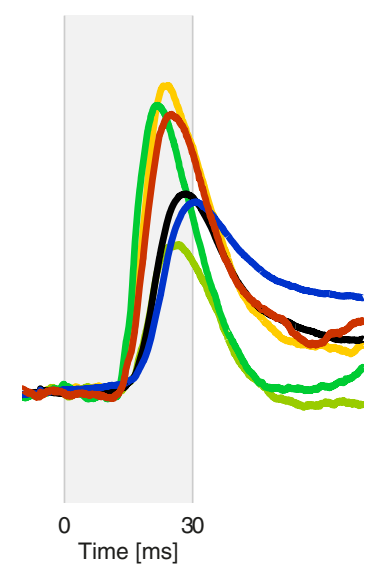

B
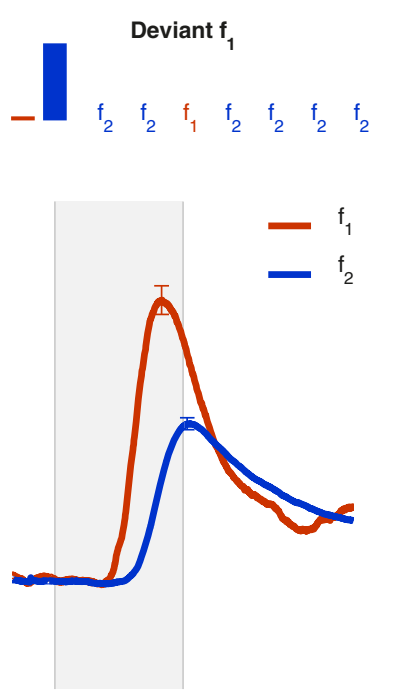

E

Diverse-broad
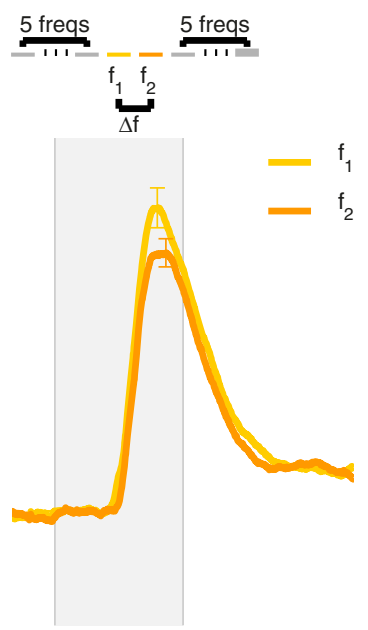

H

$f_{2}$ all conditions

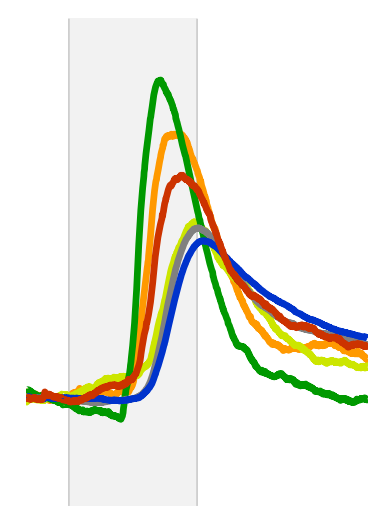

C Equal

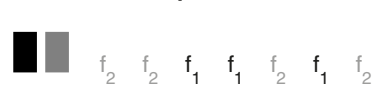

$\mathbf{F}$
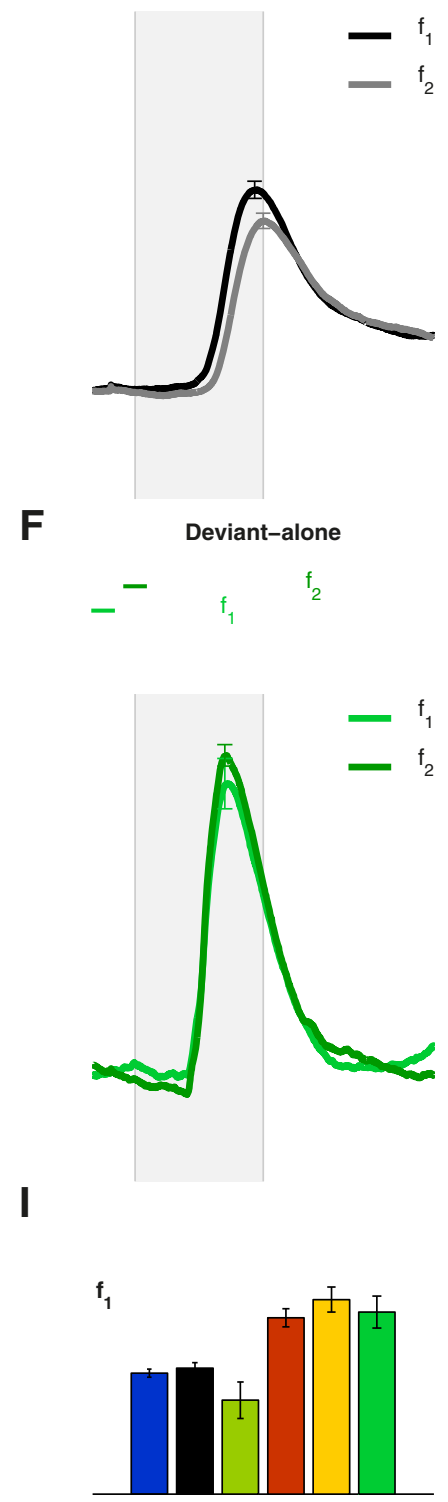

$f_{2}$

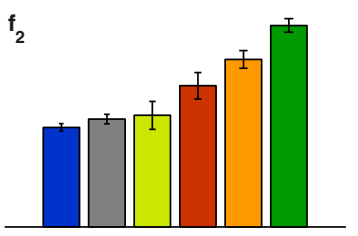

Figure 1. Effects of temporal context on the average membrane potential of a neuron in auditory cortex. This neuron did not have spiking responses. $A$, Top, A schematic representation of the Deviant $f_{2} 0$ ddball sequence in which two tones were presented randomly. In $95 \%$ of the trials, the low tone ( $f_{1}=11.8 \mathrm{kHz}$ ) was presented and in the other $5 \%$ of the trials the high tone ( $f_{2}=$ $17.1 \mathrm{kHz}$ ) was presented $(\Delta f=44 \%)$. Bottom, The average membrane potential in response to $f_{1}$ (blue) and $f_{2}$ (red). Gray background represents stimulus presentation time ( $\left.30 \mathrm{~ms}\right)$. B, Top, A schematic representation of the Deviant $f_{1}$ Oddball sequence. The low tone $\left(f_{1}\right)$ was presented in $5 \%$ of the trials, and the high tone $\left(f_{2}\right)$ was presented in $95 \%$ of the trials. Bottom, Average membrane potential in response to $f_{1}\left(\right.$ red) and $f_{2}$ (blue). $C$, Top, A schematic representation of the Equal control condition in which both $f_{1}$ and $f_{2}$ were presented in $50 \%$ of the trials. Bottom, Average membrane potential for both tones. $\boldsymbol{D}$, Top, A schematic representation of the Diverse-narrow sequence in which the main two tones, $f_{1}$ and $f_{2}$, were presented among 18 other tones randomly. The ratio between adjacent tones was $3.37 \%$. Bottom, Average response to $f_{1}$ and $f_{2}$ in this sequence. $E$, Top, A schematic representation of the Diverse-broad sequence in which the main two tones, $f_{1}$ and $f_{2}$, were presented among 10 other tones randomly. The spectral separation between each adjacent tone was $\Delta f$, the interval between $f_{1}$ and $f_{2}$ (44\% here). Bottom, Average membrane potential in response to $f_{1}$ and $f_{2} . F$, Top, A schematic representation of the Deviant-alone sequence. Each tone was presented rarely as Deviant, while all other trials were silent. Bottom, Average responses to $f_{1}$ and $f_{2}$. G, Responses to $f_{1}$ tone in all conditions. $\boldsymbol{H}$, Responses to $f_{2}$ tone in all conditions. $\boldsymbol{I}$, Peak responses to $f_{1}$ and $f_{2}$ in all conditions. freqs, Frequencies. 
zero within an interval of 1.5 times the spike width following the peak of the spike. Using these time points (determined from the filtered signal), the spikes were clipped from the unfiltered signal by replacing the spike with a straight line joining the start to the end of the spike. The clipped signal thus obtained was used in all further analyses of the membrane potential responses.

Membrane potential responses were quantified most often as the difference between the peak of the average membrane potential and the average baseline preceding stimulus onset (averaged over $5 \mathrm{~ms}$ just preceding stimulus onset). In some cases, we analyzed the peak depolarization values themselves, without subtracting the baseline (see Figs. 8, 9). Spiking responses were quantified by the peak of the smoothed $(10 \mathrm{~ms}$ hamming window) peristimulus time histogram (PSTH), without correcting for spontaneous activity, as done in previous studies both in cortex (Taaseh et al., 2011) and in subcortical stations (Malmierca et al., 2009; Antunes et al., 2010). When latency is reported, it is the interval from stimulus onset to the peak depolarization. Average responses were aligned to the average baseline computed from all trials in the corresponding block (Figs. 1, 2, 3, 4; see Figs. 6, 7).

A test of auditory responsiveness was conducted for each set. Sets that showed a significant response to the Deviant-alone sequence $(t$ test, $p<$ $0.05)$ were included in our analysis. In a few sets ( 25 of 130,3 of 48,1 of 62,3 of 42 for $\Delta f$ values of $44 \%, 21 \%, 10 \%$, and $4 \%$, respectively), the Deviant-alone sequence was missing, and the auditory responsiveness test was conducted on the Deviant response instead.

To quantify the change in response between Standard and Deviant, we use the SSA index (SI) as used previously (Ulanovsky et al., 2003):

$$
\begin{aligned}
& \mathrm{SI}_{1}=\left(d\left(f_{1}\right)-s\left(f_{1}\right)\right) /\left(d\left(f_{1}\right)+s\left(f_{1}\right)\right) \\
& \mathrm{SI}_{2}=\left(d\left(f_{2}\right)-s\left(f_{2}\right)\right) /\left(d\left(f_{2}\right)+s\left(f_{2}\right)\right),
\end{aligned}
$$

where $d\left(f_{i}\right)$ and $s\left(f_{i}\right)$ represent the responses to $f_{i}$ when it was Deviant and Standard, respectively. The common contrast between the responses characterizes the average effect of adaptation for both tones $\left(f_{1}, f_{2}\right)$ and was defined as done previously (Antunes et al., 2010; Taaseh et al., 2011):

$$
\mathrm{CSI}=\left(d\left(f_{1}\right)+d\left(f_{2}\right)-s\left(f_{1}\right)-s\left(f_{2}\right)\right) /\left(d\left(f_{1}\right)+d\left(f_{2}\right)+s\left(f_{1}\right)+s\left(f_{2}\right)\right) .
$$

For single-trial analysis (see Figs. 7, 8, 9), the membrane potential peaks were determined for each trial in the $60 \mathrm{~ms}$ time window following stimulus onset.

We fitted the adaptation model of Taaseh et al. (2011), described here in short, to our data. In the model, the presentation of tones of frequency $f$ causes some adaptation at frequency $f_{0}$, which depends on the probability of tone $f$ in the sequence and on the frequency separation between $f$ and $f_{0}$. The dependence of the amount of adaptation on the frequency separation was modeled as a Gaussian, as follows:

$$
K\left(f_{0}, f\right)=\exp \left(-\frac{\left(\log _{2} f-\log _{2} f_{0}\right)^{2}}{2 \sigma^{2}}\right) .
$$

The main parameter of interest of the model was $\sigma$, the half-width of the adaptation channel. We defined an overall adaptation load (at $\left.f_{0}\right)$ as follows:

$$
\sum_{f} p_{f} K\left(f_{0}, f\right)
$$

where the sum included all frequencies that occurred in the sequence, and each frequency contributed to the adaptation load in proportion to its probability in the sequence, $p_{f}$. Finally, the response to $f_{0}$ was postulated to be a decreasing function of the adaptation load. In the study by Taaseh et al. (2011), the following relationship was posited between the adaptation load and the response:

$$
R\left(f_{0}\right)=A \cdot B^{\Sigma_{f} p_{f} K\left(f_{0}, f\right)} .
$$

Here $A$ is the unadapted response, and $B<1$ measures the strength of the adaptation. Taaseh et al. (2011) have also solved a model analytically with depletion of synaptic resources and exponential recovery, showing that the decreasing function relating the adaptation load to the response was somewhat different from the exponential form used here. Nevertheless, the exponential form captured the same qualitative behavior as the exact solution and had significant numerical advantages. It was therefore kept here in the same form used for similar calculations by Taaseh et al. (2011).

The model has the following three parameters: $A, B$, and $\sigma$. The modeled data were always normalized by the responses in the Deviant-alone condition. In consequence, $A$ was generally close to 1 . The parameter $B$ was determined primarily by the relative size of the responses to the tone when Standard (for more details, see Taaseh et al. (2011)). Thus, the most interesting parameter of the model is $\sigma$, which determines the relative size of the responses in the other conditions, primarily the Deviant and the Diverse-broad conditions.

The model was first fitted to the average responses to all conditions at all $\Delta f$ values tested for each neuron separately. For analyzing the consistency of the responses across conditions, we also fitted models to subsets of the responses in which each test condition was removed in its turn from the training set, and compared the predicted responses of these models with the measured responses to the condition that was left out.

Models were trained (separately in each neuron) by minimizing the overall error of the model, $\varepsilon^{2}$, which was defined as follows: $\varepsilon^{2}=\Sigma_{c} w_{c}\left(r_{c}-m_{c}\right)^{2}$, where the sum was over the six conditions in which each tone was tested and over all $\Delta f$ values, $r_{c}$ is the measured response in each condition, $m_{c}$ is the model prediction, and $w_{c}$ is a weight, which was taken to be the square root of the number of stimulus presentations at that condition. Given $\sigma, \varepsilon^{2}$ was minimized to produce optimal $A$ and $B$ (using the fit function from the Matlab curve-fitting toolbox). We then performed a one-dimensional search on $\sigma$ to minimize $\varepsilon^{2}$. We constrained $\sigma$ to be between 0.05 and 4 octaves. To quantify the fit of the model to the data, we compared the mean error $\left(\varepsilon^{2}\right)$ with the weighted sum of squared SEs of the mean responses: $s e^{2}=\Sigma_{c} w_{c} s e_{c}^{2}$, where the sum was over the same conditions as $\varepsilon^{2}, w_{c}$ denotes the same weights used in calculating $\varepsilon^{2}$, and $s e_{c}$ denotes the SE of $r_{c}$. This number represents an estimate of the mean error of the best possible model for the data, which has as many parameters as conditions, and which estimates the response to each condition by its observed mean.

\section{Results \\ The Oddball paradigm}

Figure 1 shows responses of one neuron to the same two tones ( $f_{1}=11.8 \mathrm{kHz}, f_{2}=17.1 \mathrm{kHz}, \Delta f=44 \%$, ISI $\left.=300 \mathrm{~ms}\right)$ embedded in all types of sequences (see Materials and Methods). The top panel of Figure $1 \mathrm{~A}$ represents schematically the Deviant $f_{2}$ block in which $5 \%$ of the stimuli had $f_{2}$ and $95 \%$ had $f_{1}$, presented in a pseudorandom order. The bottom panel of Figure $1 A$ shows the average membrane potential in response to the two tones. The resting potential of this neuron was rather low $(-80 \mathrm{mV}$ at baseline), and sensory stimulation elicited only subthreshold activity with no spiking. Tone pips at $f_{2}$, the Deviant, elicited on average a larger sensory EPSP than tone pips at $f_{1}$, the Standard. To verify that these differences emerge from sensitivity to stimulus probability and not from frequency selectivity, we switched the roles of $f_{1}$ and $f_{2}$, as illustrated schematically in the top of Figure $1 B$ (Deviant $f_{1}$ block). In this case, the average response to $f_{1}$ was larger than the response to $f_{2}$, reversing the order of the responses in Figure $1 A$. The contrast between the response to the same tone when it was Standard and when it was Deviant (i.e., the SI) was 0.19 for $f_{1}$ and 0.17 for $f_{2}$, both revealing specific adaptation of the responses to the respective Standard.

A third block was presented to compare the responses when the two tones presented in equal probability ( 250 trials with each frequency, pseudorandomly; Fig. $1 C$, top). In this condition, the average response was typically larger than the response of the same tone when Standard and smaller than the response of the same tone when Deviant [Figs. 1G-I; red (Deviant), black/gray (Equal), blue (Standard)]. 

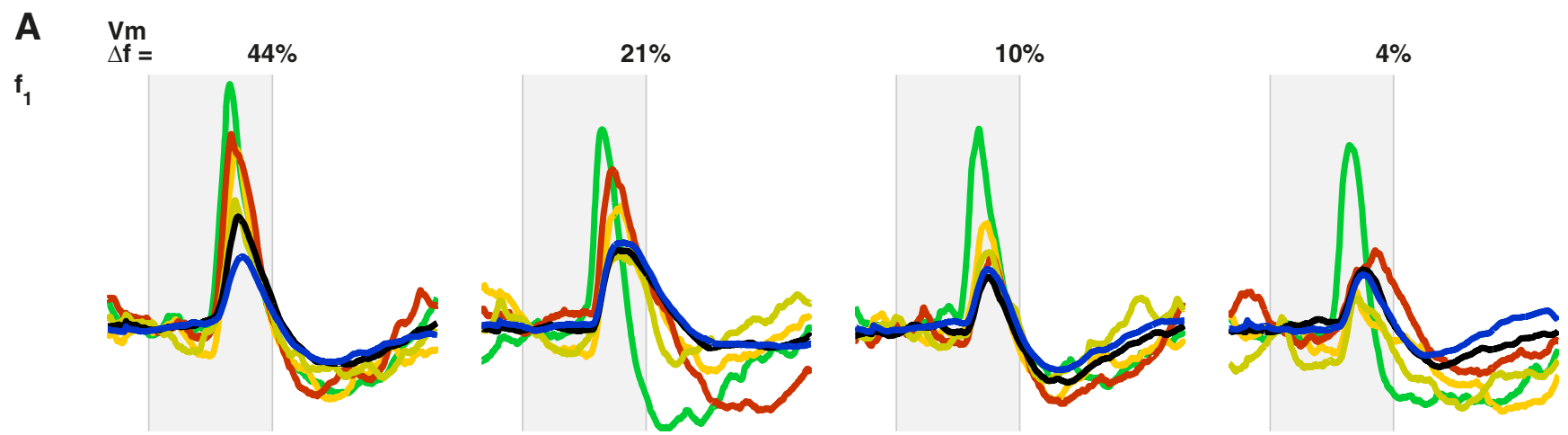

$f_{2}$
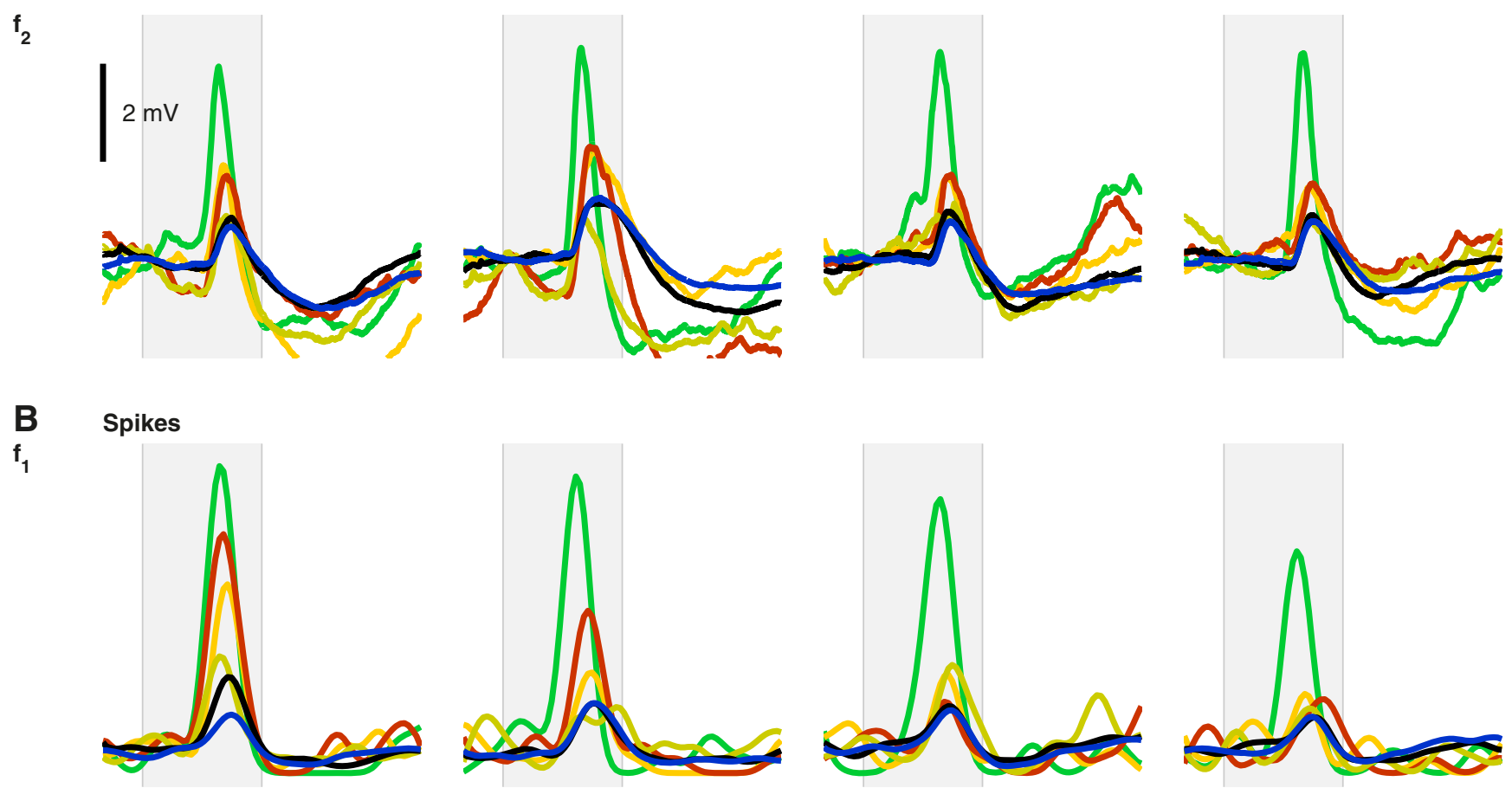

$f_{2}$
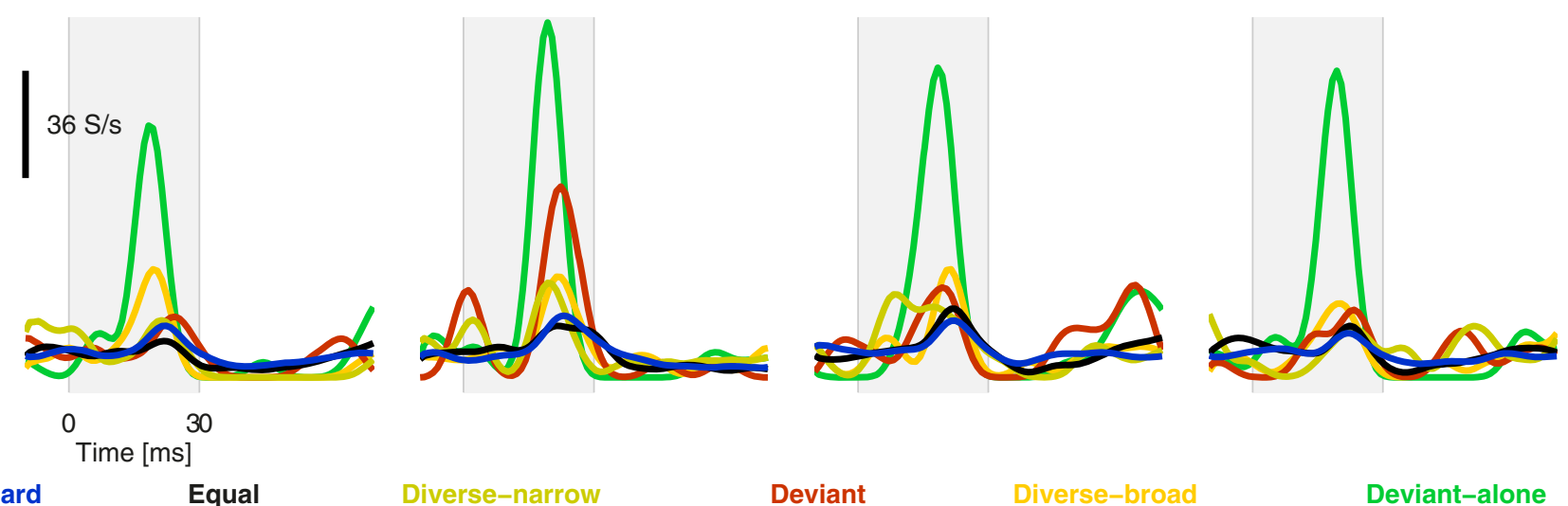

Standard

Equal

Diverse-narrow

Deviant

Diverse-broad

Deviant-alone

Figure 2. Responses of a typical neuron to $f_{1}$ and $f_{2}$ in all conditions for $\Delta f=4 \%, 10 \%, 21 \%$, and $44 \%$ (color legend in bottom panel). Membrane potential responses were calculated after spike clipping. $A$, Average membrane potential responses. $B$, Smoothed PSTHs for the spiking responses of the same neuron. Same display and color conventions as in Figure 1.

\section{The control sequences}

We used several control sequences to study the mechanisms, which may contribute to the dependence of the response on stimulus probability. These controls are the same as those used by Taaseh et al. (2011).

Figure $1 D$ (top) represents schematically the Diverse-narrow sequence (see Materials and Methods), and the Figure $1 D$ (bot- tom) shows the average membrane potential (same neuron as in Fig. $1 A-C$ ) in response to $f_{1}$ and $f_{2}$ embedded in this sequence. In this example, $\Delta f$ was $44 \%$ and the frequency ratio between adjacent frequencies of the Diverse-narrow sequence was 3.37\%. Figure $1 E$ illustrates the Diverse-broad sequence, and shows the responses of the same neuron to $f_{1}$ and $f_{2}$ embedded in this sequence. The wider frequency separation between tones in this 
A<smiles>[As]=[W]</smiles>

B

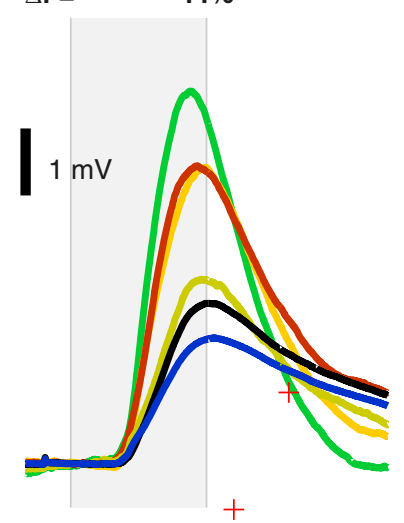

$$
\begin{aligned}
& 0 \quad+30 \neq \\
& \text { +Timte [ms] } \underset{\neq}{\neq}
\end{aligned}
$$

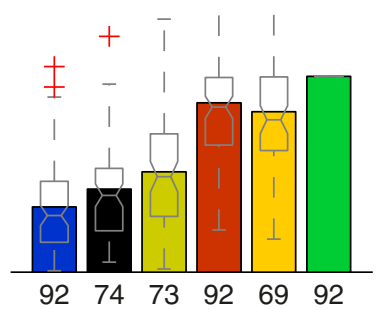

C

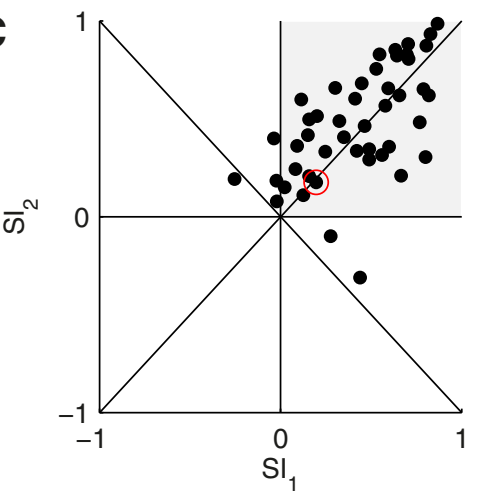

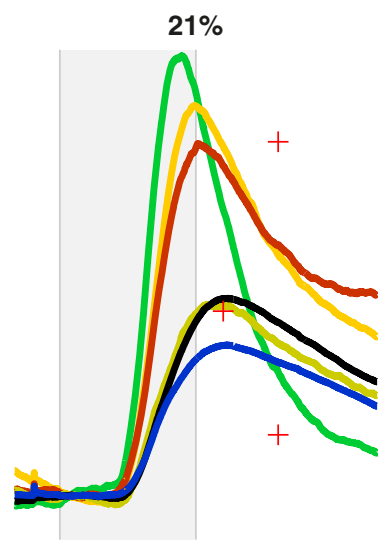
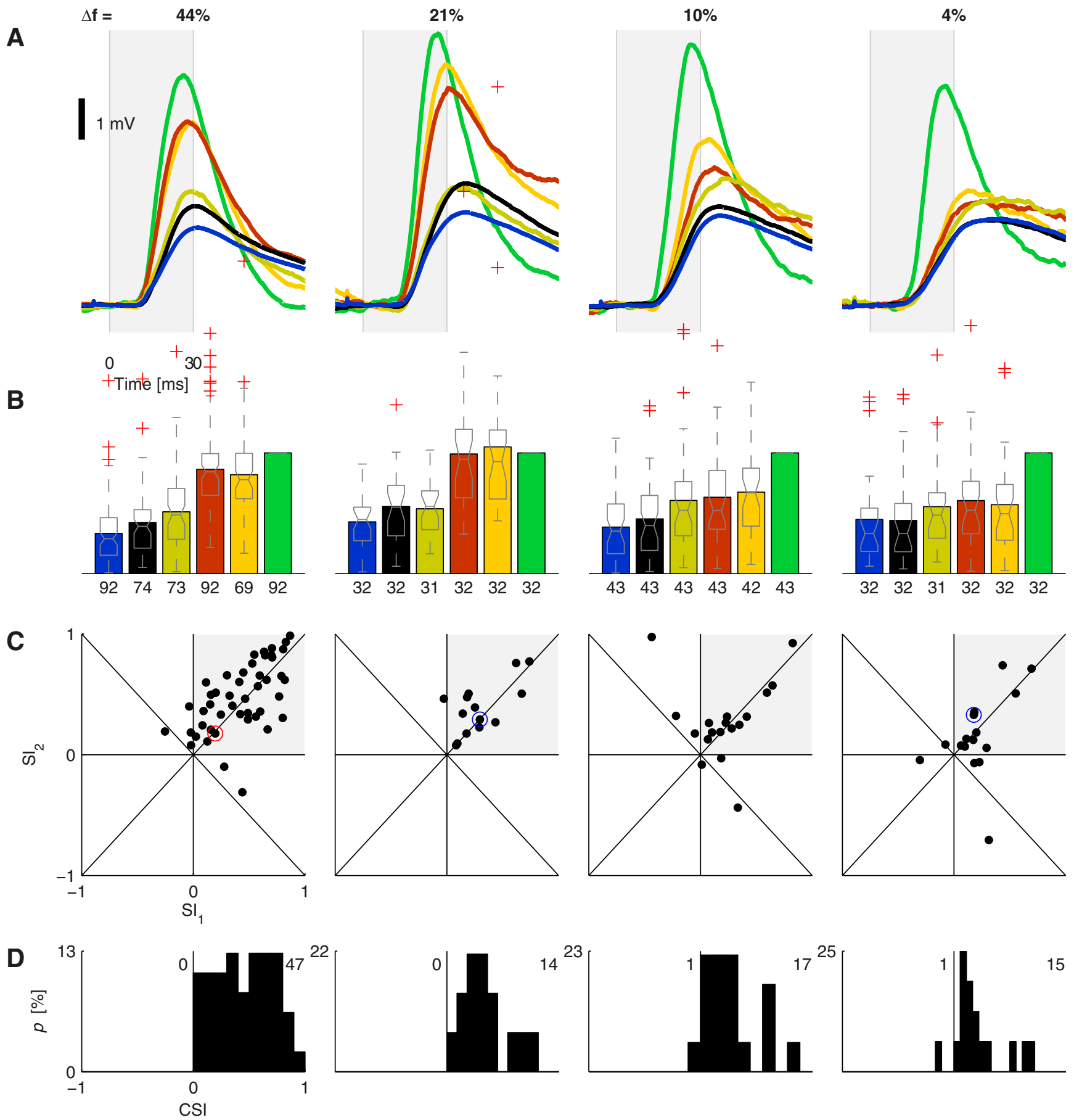

Equal

Diverse-narrow

Deviant

Diverse-broad

Deviant-alone

Figure 3. A, Population membrane potential responses $(n=55)$. $\boldsymbol{B}$, Average normalized peak responses (bars) and a rough outline of their distributions (displayed as box plots) for the data as in $\boldsymbol{A}$. The responses were normalized to the responses evoked in the Deviant-alone sequence of the each set. The number of responses in each bar is displayed below. $C$, $\mathrm{SI}_{1}$ and $\mathrm{SI}_{2}$ for each neuron plotted against each other. Red circle in $\Delta f=44 \%$ marks responses for the neuron in Figure 1. Blue circles in $\Delta f=21 \%$ and $4 \%$ mark the neuron that is shown in Figure 2 . This neuron is not marked in $44 \%$ and $10 \%$ since its auditory responses were only marginally significant for one of the tones and therefore were not included in the corresponding scatter plots. D, Distribution of the CSI as a function of $\Delta f$.

sequence (see Materials and Methods) resulted in less overall adaptation and, therefore, in larger responses than in the Diversenarrow sequence. The last control was the Deviant-alone sequence, in which the Deviant was presented randomly 25 times as in the Oddball paradigm (5\%), while the other 475 trials did not include any stimulus. Figure $1 F$ illustrates schematically these sequences and displays the average response for $f_{1}$ and $f_{2}$ in these blocks. The long time interval between successive sound presentations in the Deviant-alone sequences was expected to lead to full recovery of the responses to tones in these sequences, and indeed the responses in Figure $1 F$ were the largest among all sequences.

Figure $1, G$ and $H$, replots the average responses to the two frequencies from Figure $1 A-F$, separately for $f_{1}$ and $f_{2}$. The peak 
A

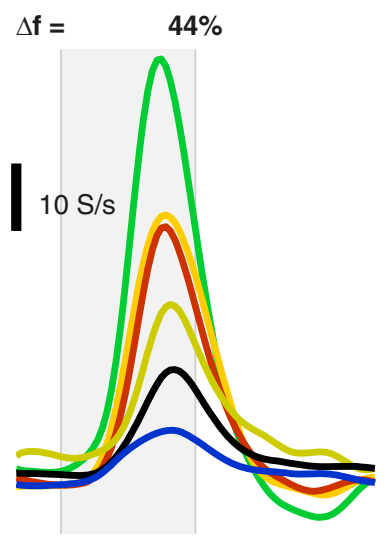

0
$21 \%$

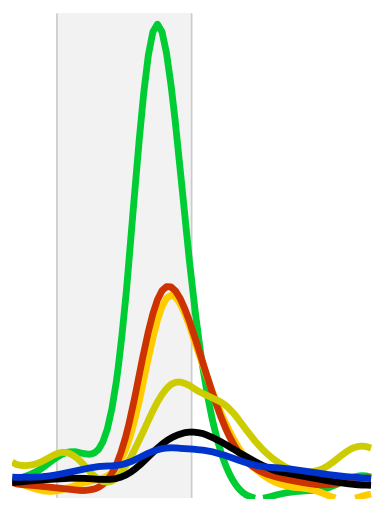

$10 \%$

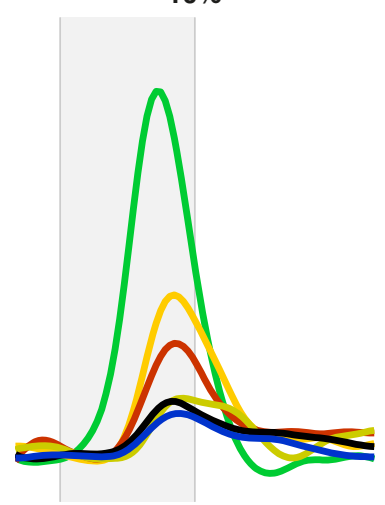

$4 \%$

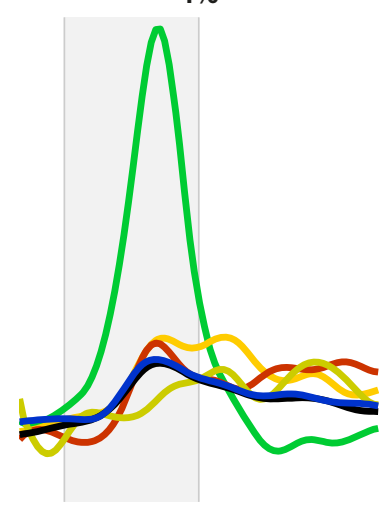

B
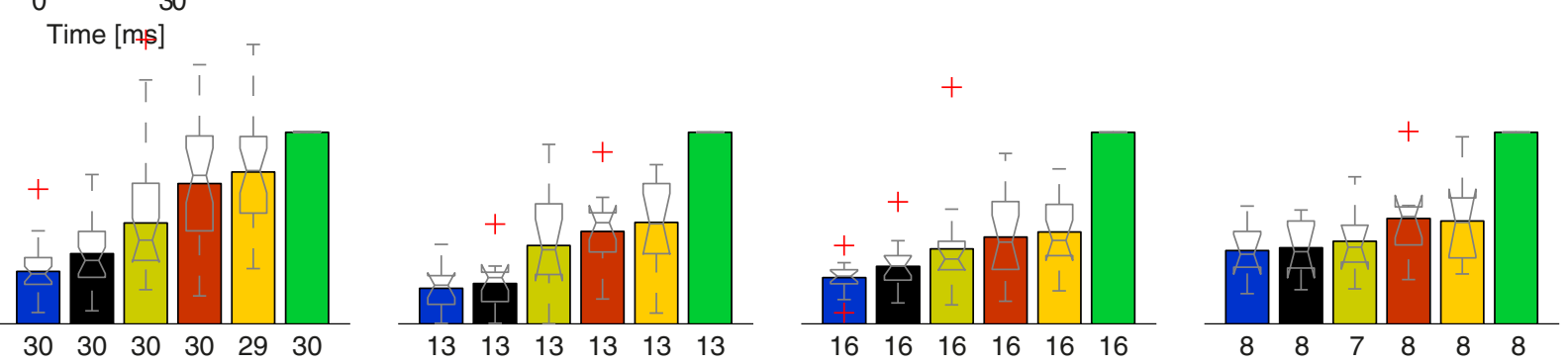

C
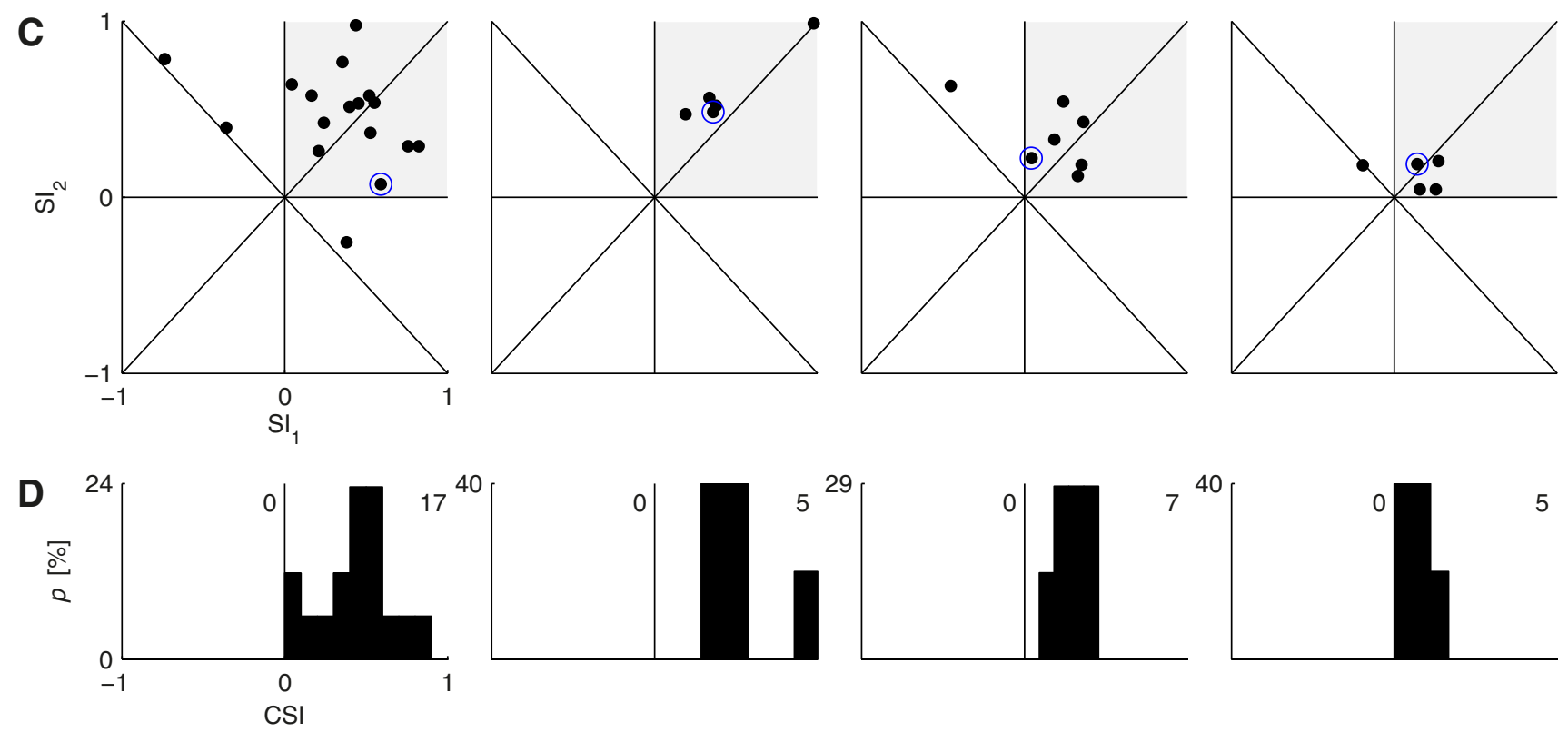

Standard

Equal

Diverse-narrow

Deviant

Diverse-broad

Deviant-alone

Figure 4. Population summary for spiking responses, using the same format as in Figure 3. A, Average population response for spiking activity represented as smoothed PSTHs. B, Average normalized peak responses (bars) and a rough outline of their distributions (displayed as box plots). The responses were normalized to the responses evoked in the Deviant-alone sequence of the each set. C, SI1 and SI2 for each neuron plotted against each other. Blue circles mark the neuron shown in Figure 2. D, Distribution of the CSI as a function of $\Delta f$.

response (i.e., maximum value of the average response - average baseline value) is presented in Figure 1I. This neuron responded more weakly to the Standard, Equal, and Diverse-narrow conditions of both frequencies, and responded more strongly in the Deviant, Diverse-broad, and Deviant-alone conditions. Thus, the same tone elicited weaker responses in sequences in which it was repeated often (Standard, Equal) or when it was presented as part of a dense set of frequencies (Diverse-narrow), suggesting the presence of crossfrequency adaptation. Larger responses were elicited by the same tones when presented rarely, and when other tones in the sequence were spectrally widely separated (as in the Deviant and Diversebroad conditions). As a rule, the stronger responses had somewhat shorter average latencies. Because this dependence was almost universal, we analyze here in details only the response magnitude.

\section{SSA is present at all tested $\Delta f$}

Neurons were tested with similar sequences that had different $\Delta f$ values (44\% as in Fig. 1, 21\%, 10\%, and 4\%). Figure 2 shows 
typical average responses of one neuron to the six sequences at all tested $\Delta f$ values for membrane potential activity (Fig. $2 A$ ) and smoothed PSTHs based on its spiking responses (Fig. $2 B$ ). For each $\Delta f$ value, a different pair of frequencies was used $\left(f_{1}\right.$ and $\left.f_{2}\right)$, but the center frequency of all pairs $\left(\sqrt{f_{1} \times f_{2}}\right)$ was the same and consisted of the approximate BF of this neuron $(5.6 \mathrm{kHz})$.

Consider first the Deviant-alone responses. In these sequences, tones of only one frequency were presented, but very rarely. In consequence, the value of $\Delta f$ did not matter-the responses are all approximately the same, consistent with the frequency tuning of this neuron, which was wider than the largest $\Delta f$ used here at the sound level used (data not shown). Similarly, the responses to Standards did not vary much with $\Delta f$, presumably because in the sequences in which a frequency is Standard, most of the reduction of the response was due to the effects of the large probability of that frequency, and the few Deviant presentations played a minor role in determining the responses to the Standard.

In contrast, the responses in the Deviant and Diverse-broad conditions generally decreased with decreasing $\Delta f$, presumably because the other frequencies that appeared in the corresponding sequences got closer to one another and, consequently, adapted the responses to each other. This observation will be quantified in details later. For the neuron in Figure 2, the responses to $f_{1}$ indeed decreased when $\Delta f$ decreased, but for $f_{2}$ the response for $\Delta f=44 \%$ was smaller than for $\Delta f=21 \%$. This was due to the fact that at $\Delta f=44 \%, f_{2}$ was at the edge of the tuning curve, and with $\Delta f=21 \%$ it was closer to the BF. Nevertheless, the relative order of the responses remained pretty much the same at all $\Delta f$ values: Deviant-alone responses were largest, Deviant and Diverse-broad elicited comparable responses, and Standard was smallest, with Equal and Diverse-narrow responses mostly similar to those evoked by the same frequency when Standard. Similar to the neuron in Figure 1, larger responses tended to have shorter latencies.

The pattern of responses illustrated by examples in Figures 1 and 2 was typical. Figures 3 and 4 summarize the average membrane potential and spike responses for the whole dataset. Figures $3 A$ and $4 A$ show the average responses calculated using all sets that showed significant auditory responses for both $f_{1}$ and $f_{2}$ (membrane potential: $\Delta f$ of $44 \%, 21 \%, 10 \%$, and $4 \%, 47$ of 65,14 of 25,18 of 31 , and 16 of 21 cases; Spikes: $\Delta f$ of $44 \%, 21 \%, 10 \%$, and $4 \%, 17$ of 22,5 of 10, 7 of 13 , and 5 of 8 cases). Figures $3 B$ and $4 B$ show the average responses normalized to the Deviant-alone condition (bars, only for sets that had significant Deviant-alone sequences) and a rough outline of their distributions (displayed as box plots) for each of the conditions. The relative order of the responses, averaged over all neurons, was very similar at all $\Delta f$ values. Deviant-alone responses were, on average, the largest. Diverse-broad and Deviant responses came next, with the two having essentially the same average at all $\Delta f$ values. The Diversenarrow sequence was next, except for the average membrane potential responses with $\Delta f=21 \%$ (Fig. $3 B$ ), where Equal responses were slightly larger on average. Standards evoked the smallest responses. Slight inconsistencies in the relative order of the averaged responses (Figs. $3 A, 4 A$ ) and in the average of the peak responses (Figs. $3 B, 4 B$ ) can be observed, for example, in the membrane potential responses with $\Delta f=21 \%$, where the Deviant-alone condition was the largest in Figure $3 A$, but was slightly smaller than the Deviant and Diverse-broad in Figure $3 B$. Such discrepancies are due to the different way in which the responses were normalized before averaging.

As illustrated in Figures 1, 2, and 3, larger responses tended to have shorter latencies. Thus, the average latencies to peak of the Deviant responses were shorter on average [28.6 $\pm 10.9,34.9 \pm$
$13.7,36 \pm 13.1$, and $41.5 \pm 17.8 \mathrm{~ms}( \pm \mathrm{SD})$ for $\Delta f=44 \%, 21 \%$, $10 \%$, and $4 \%$, respectively] than Standard latencies (35.2 \pm 13.1 , $37.6 \pm 11.5,41 \pm 15.8$, and $42.3 \pm 15.4 \mathrm{~ms}$ ). Note, however, that the differences in peak latencies were small relative to the whole duration of the response, which could span tens of milliseconds (Figs. 3, 4), as well as relative to the scatter of the peak responses among neurons, and the difference between Standard and Deviant latencies was significant only for $\Delta f=44 \%[\Delta f=44 \%$ : $p<$ $0.00005, t=5.8, \mathrm{df}=107 ; \Delta f=21 \%: p=0.23, t=1.2, \mathrm{df}=33$; $\Delta f=10 \%: p=0.03, t=2.2, \mathrm{df}=43 ; \Delta f=4 \%: p=0.18, t=0.23$, $\mathrm{df}=34$ (paired $t$ test)].

Remarkably, even for $\Delta f=4 \%$, Deviant responses were significantly larger than the responses to the Standard for both membrane potential and spikes [membrane potential: $t=3.1$, $\mathrm{df}=31, p=0.004$; spikes: $t=4.3, \mathrm{df}=7, p=0.0035$ (paired $t$ test)]. This $\Delta f$ is near the behavioral frequency discrimination limen of the rat (Talwar and Gerstein, 1998; Sloan et al., 2009).

Figures $3 C$ and $4 C$ compare the SIs calculated for the two frequencies, for each neuron separately (see Materials and Methods). For most neurons, both $\mathrm{SI}_{1}$ and $\mathrm{SI}_{2}$ were positive, both for membrane potential and spike data. Thus, in the large majority of the neurons, the responses to a frequency when Deviant tended to be larger than the responses to the same frequency when Standard, independently of the other frequency used in the Oddball sequences. Furthermore, there was no preference for the high $\left(f_{2}\right)$ or the low $\left(f_{1}\right)$ tone, since the data points in Figures $3 C$ and $4 C$ are approximately equally distributed above or below the diagonal.

The common SI (CSI, see Materials and Methods) characterizes the average effect of adaptation for both $f_{1}$ and $f_{2}$. Figures $3 D$ and $4 D$ present the CSI in all $\Delta f$ values for all the neurons shown in Figures $3 C$ and $4 C$, respectively. The CSIs were positive for the spiking responses at all conditions. They were also all positive for the membrane potential responses when tested with the larger $\Delta f$ values $(21 \%$ and $44 \%$ ). For the smaller $\Delta f$ values ( $4 \%$ and $10 \%)$, some small negative CSI values were observed for membrane potential responses, but the CSI was significantly larger than zero on average at all conditions (Sign test: $p_{4 \%}=0.0005, p_{10 \%}=0.0001$ ).

\section{Dependence of SSA on ISI}

The large responses in the Deviant-alone condition suggests the presence of a time-dependent recovery process. Thus, longer interstimulus time intervals were expected to lead to less adaptation of the responses to Standards and therefore to less SSA overall. Figure $5 \mathrm{~A}$ displays the mean membrane potential responses of one neuron (averaged over $f_{1}$ and $f_{2}$ ) to Standard, Deviant, and Deviant-alone (same color code as in Figs. 1, 2, 3, 4) for 3 ISIs (300, 700, and 1200 $\mathrm{ms})$. As expected, longer ISIs were associated with stronger responses. The responses to Standards showed a particularly strong dependence on ISI, resulting in lower contrast between Standard and Deviant responses at longer ISIs. Figure $5 B$ shows the SIs for membrane potential responses recorded with the three ISIs (the data for $300 \mathrm{~ms}$ is the same as in Fig. 3C; fewer data were collected for 700 and $1200 \mathrm{~ms}$ ). Similar to the neuron in Figure 5A (blue circle), SSA decreases for longer ISIs. Nevertheless, significant SSA occurred for ISIs as long as $1200 \mathrm{~ms}$. This result supports the major role of intracortical synaptic mechanisms, which recover at about this time scale, rather than that of purely thalamic mechanisms, which recover faster (Wehr and Zador, 2005).

\section{SSA is transformed by cortical neurons}

While the membrane potential represents the summed inputs to the cortical neurons, the spikes represent their output. Figure $6 \mathrm{~A}$ compares the CSI of membrane potential responses $\left[\mathrm{CSI}\left(V_{\mathrm{m}}\right)\right]$ 


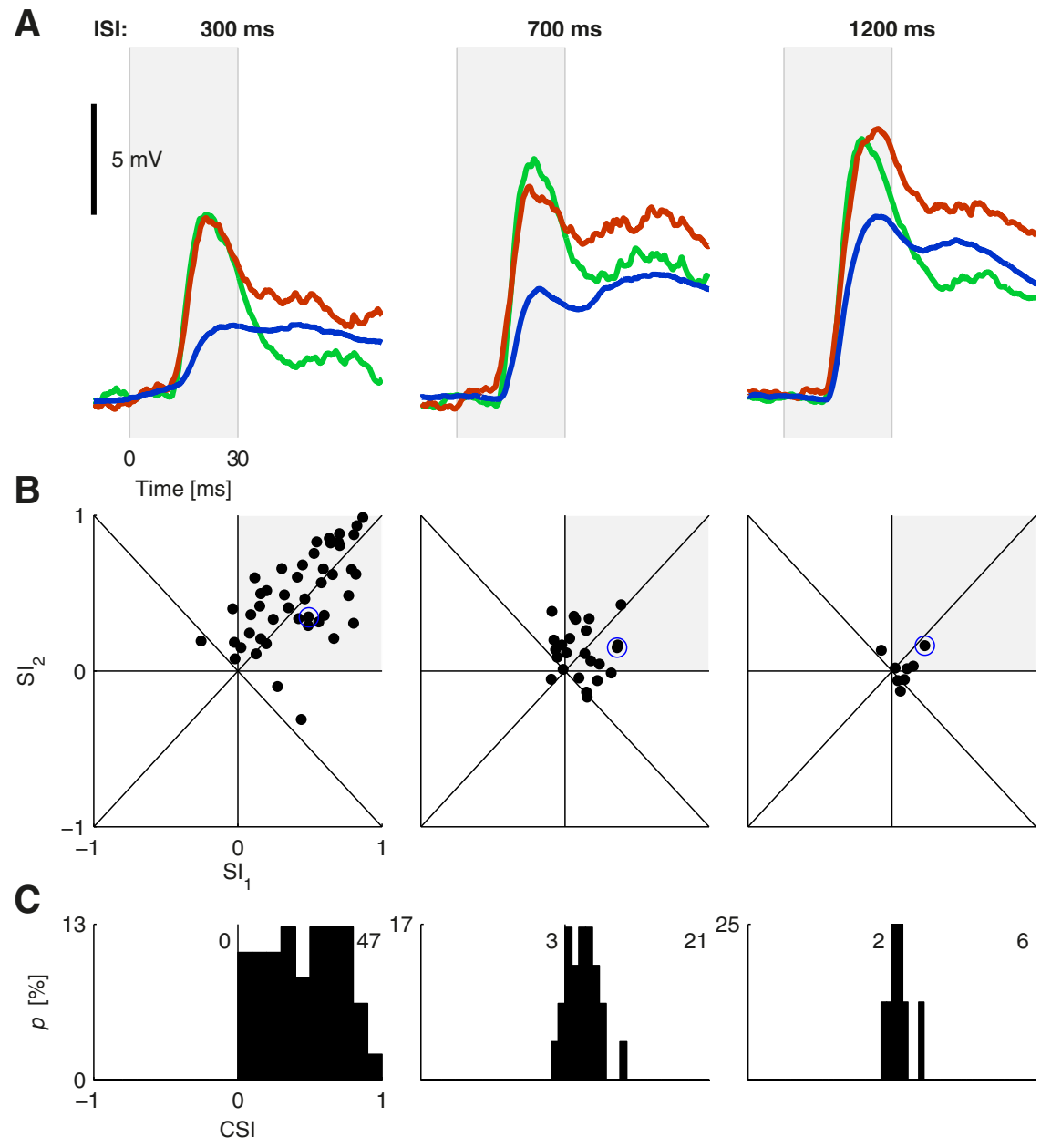

Figure 5. $\quad \boldsymbol{A}$, Membrane potential responses of a neuron in Standard (blue), Deviant (red), and Deviant-alone (green) conditions for three different ISIs (300, 700, and $1200 \mathrm{~ms}$, averaged over $f_{1}$ and $\left.f_{2}, \Delta f=44 \%\right)$. $\boldsymbol{B}, \mathrm{SI}_{1}$ and $\mathrm{SI}_{2}$ for each neuron plotted against each other for all neurons tested with the three ISIs as in $\boldsymbol{A}(\Delta f=44 \%)$. For ISI $=300$, the plot is the same as in Figure 3 and is replotted for comparison. Blue circles mark the neuron that is shown in $\boldsymbol{A}$. C, Distribution of the CSI for the different ISIs.

and spiking responses [CSI(Spk)] in neurons that had significant auditory responses with both signals. For all $\Delta f$ values, the CSI(Spk) (ordinate) could be larger or smaller than that computed from the $\operatorname{CSI}\left(V_{\mathrm{m}}\right)$ (abscissa) responses.

However, the CSIs were calculated in a subtly different way for spikes and for membrane potentials. For the membrane potential responses, we quantified the responses by the increase in membrane potential with respect to the average baseline. On the other hand, for spikes, we quantified the responses by their peak (not correcting for the baseline firing rates of the neurons), as has been done in previous studies (Ulanovsky et al., 2003; Malmierca et al., 2009; Antunes et al., 2010; Taaseh et al., 2011). Neurons with higher spontaneous firing rates tended therefore to have lower CSI values for their spiking responses because the denominator of the CSI could include a significant contribution of the spontaneous rate. We verified this effect by plotting the differences between $\operatorname{CSI}\left(V_{\mathrm{m}}\right)$ values and $\operatorname{CSI}(\mathrm{Spk})$ values $\left[\Delta \operatorname{CSI}=\operatorname{CSI}\left(V_{\mathrm{m}}\right)-\operatorname{CSI}(\mathrm{Spk})\right]$ against the baseline firing rate of each neuron (Fig. $6 B$ ). Higher spontaneous rates corresponded generally to larger values for $\Delta$ CSI [presumably because of the smaller CSI(Spk) due to the effect of the spontaneous rate].

To get a better comparison between the level of SSA at both signals, we therefore modified the calculation of CSI(Spk) to make it more comparable to $\operatorname{CSI}\left(V_{\mathrm{m}}\right)$ by subtracting the spontaneous rate from the peak spiking responses before calculating the CSI. The modified CSI(Spk) was almost uniformly larger than $\operatorname{CSI}\left(V_{\mathrm{m}}\right)$ (Fig. $6 C)$. Furthermore, the modified $\Delta \mathrm{CSI}$ [defined as above, using the baselinecorrected firing rates to calculate CSI(Spk)] lost much of its correlation with the baseline firing rate (Fig. $6 D$ ).

These effects are illustrated by specific examples in Figure 7. The responses of the neuron marked in Figure $6 A$ with a red circle above the main diagonal of the $\Delta f=$ $44 \%$ panel are presented in Figure 7, A1, $B 1$, and $D 1$. The left column in Figure 7A1 shows the average membrane potential for the two tones $\left(f_{1}, f_{2}\right)$ in the six sequences. The right column in Figure 7A1 shows the smoothed PSTHs for the spiking responses of the same neuron. The peak responses are displayed in Figure $7 B 1$. Even without correcting for spontaneous firing rate, the contrast between the membrane potential responses to Standard and Deviant tones $\left(\mathrm{SI}_{1}=0.42, \mathrm{SI}_{2}=\right.$ 0.34 , CSI $=0.39)$ was similar or lower than the contrast in its spiking activity (especially for $f_{1}, \mathrm{SI}_{1}=0.76, \mathrm{SI}_{2}=0.29$, $\mathrm{CSI}=0.65)$. The modified CSI $=0.79$ was even larger, as expected. Figure 7D1 displays the spiking responses as a raster plot. This neuron had a very low spontaneous activity. A plausible explanation for the increased contrast between Standards and Deviants is an "iceberg effect" (Carandini and Ferster (2000)). The term iceberg effect refers to the relationships between membrane potential and spiking responses that are due to the presence of spiking threshold: sensory-driven changes in the membrane potential (the iceberg) lead to spiking only when large enough, increasing the contrast between the small and large responses. In the particular case of the Oddball sequences, the iceberg effect could increase the contrast between the responses to Standards (which may fail to cross the threshold at all) and the larger responses to Deviants (Pérez-González and Malmierca, 2012). To check for the presence of an iceberg effect, Figure $7 C 1$ compares the normalized spiking and membrane potential responses to all conditions available for this neuron (12 conditions: $1 \Delta f, 2$ tones, 6 conditions, responses normalized to the average Deviant-alone response). This relationship was fitted better by an exponential (continuous line, mean square root error $=0.14$ ) than by a straight line (dashed line, mean square root error $=0.23$ ). The approximate exponential dependence of the spiking responses on the membrane potential is consistent with the effect of a threshold on the firing rates and is most probably the cause of the iceberg effect.

A neuron whose $\operatorname{CSI}(\mathrm{Spk})$ was smaller than $\operatorname{CSI}\left(V_{\mathrm{m}}\right)$ is marked with a red circle below the diagonal of the $\Delta f=44 \%$ panel in Figure 6A, and its responses are displayed in Figure $7 A 2-D 2$. Without correcting for the spontaneous firing rate, the membrane potential showed a slightly higher $\mathrm{SI}\left(\mathrm{SI}_{1}=0.63, \mathrm{SI}_{2}=\right.$ 

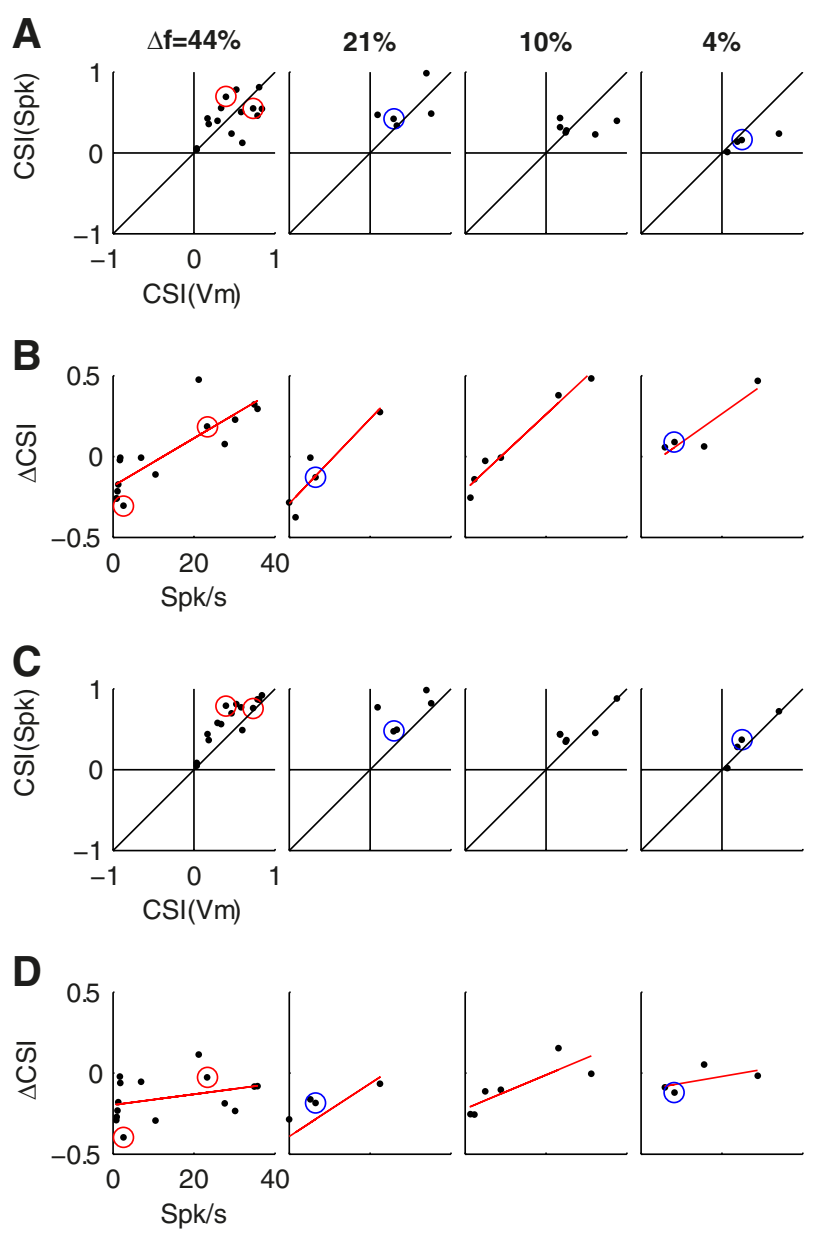

Figure 6. Comparing SSA in membrane potential and spiking responses. $A, C S I\left(V_{m}\right)$ responses against $\mathrm{CSI}(\mathrm{Spk})$ responses for all neurons that showed significant auditory responses for both. Blue circles mark the neuron that is presented in Figure 2. Red circles mark the examples used in Figure 7. B, $\Delta C S I\left[C S I\left(V_{m}\right)-C S I(S p k)\right]$ plotted against the baseline spiking rate of the neuron. Generally, a higher spontaneous rate corresponded to a larger $\Delta C S I$ (red lines linear regression lines). $C, C S I\left(V_{m}\right)$ plotted against the modified $C S I(S p k)$ (see main text for details). Red and blue circles are as in A. D, $\Delta$ CSI [calculated for the modified CSI(spk)] plotted against the baseline spiking rate. At $\Delta f=20 \%$, one dot is outside the limits of the plot $(\Delta \mathrm{CSI}=-0.67 ; \mathrm{Spk} / \mathrm{s}=1.6)$.

$0.85, \mathrm{CSI}=0.73)$ than the spiking activity $\left(\mathrm{SI}_{1}=0.52, \mathrm{SI}_{2}=0.58\right.$, $\mathrm{CSI}=0.55)$. In contrast with the first neuron shown in Figure 7 (left), this neuron had a substantial level of spontaneous activity, and changes in its membrane potential corresponded to changes in firing rates in a more linear fashion, as shown in Figure 7C2 (fitted to 36 conditions available from $3 \Delta f$ values; mean square root error $=0.23$ for the linear fit; mean square root error $=0.22$ for the exponential fit). However, even this neuron had a modified CSI for the spiking responses (0.75) that was larger than the $\operatorname{CSI}\left(V_{\mathrm{m}}\right)$, and, here too, this increase can be traced to the remaining convexity of the relationship between membrane potential and firing rates (Fig. 7D2).

\section{Single-trial responses}

At this point, we have shown that the responses to Standard tones are on average smaller than the responses to Deviant tones. This result could be due to a uniform decrease in the size of the EPSPs evoked by Standards relative to those evoked by Deviants. Alter- natively, the range of EPSP sizes could be the same for Standards and Deviants, but Deviants could have a larger probability to elicit larger EPSPs. Figure 8 examines this issue in the same neuron that was presented in Figure 1. Figure $8 A$ shows 25 randomly selected trials (light color) in the Standard condition as well as all trials in the Deviant and Deviant-alone conditions (also 25 trials each) for the two frequencies ( $f_{1}$ and $f_{2}$ ) with $\Delta f=44 \%$, together with the average response in each condition (dark color). Figure $8 B$ shows the peak membrane potential of each trial plotted against the baseline membrane potential just before stimulus onset of the same trial for all trials of the same three conditions. Both plots illustrate the finding that while the averages of the peak membrane potential in the three conditions were clearly different, the distributions of the single-trial peak responses overlapped significantly. In fact, some standard trials had peak responses as large as those of single trials in the Deviant and Deviant-alone conditions, and this was true for essentially all values of the baseline. Thus, for this neuron, the Deviant and Deviant-alone responses sampled the high end of the distribution of peak responses of Standard trials. Similarly, the distribution of peak response latencies of the different conditions overlapped to a great extent (data not shown).

The range of possible peak responses for this neuron depended on the baseline membrane potential just preceding stimulus onset (Fig. $8 B$ ). When the baseline potential was relatively low, there was a large range of possible single-trial peak responses, while for high baseline potentials the range of possible peak responses was much narrower. In fact, the upper bound of peak responses depended very weakly, if at all, on the baseline membrane potential, resulting in a wedge-shaped scatter. This finding is consistent with the notion that the peak responses were limited by the sensory reversal potential (Crochet et al., 2011). The reversal potential of the sensory input depends on the exact mixture of excitation and inhibition that is evoked by the stimulus, and is therefore a joint property of the neuron and of the applied stimulus. For this neuron, the presumed reversal potential was hyperpolarized relative to the spiking threshold. Since the sensory EPSP was limited by the reversal potential, no spikes occurred in response to tone stimulation. The finding that many neurons in sensory cortices show such a lack of spiking responses is ubiquitous (as first documented by Margrie et al. (2002). More recently, neurons whose spiking threshold was above the reversal potential of the sensory input have been studied in somatosensory cortex of awake mice by Crochet et al. (2011) and Sachidhanandam et al. (2013). Our observation of a maximal possible depolarization level for tone responses, independent of the baseline membrane potential, is consistent with their findings.

Figure $9 \mathrm{~A}$ shows similar data for six additional neurons that did not emit any spikes. For simplicity, only trials in which the lower of the two frequencies was presented are used in these plots. The plots display the peak responses in each single trial against the baseline membrane potential just preceding stimulus onset (Fig. $8 B$ ) for Standard trials (light and dark blue) and Deviant trials (red). They all show the wedge-shaped scatter, consistent with the limiting effect of a sensory reversal potential. In addition, in all examples, the Deviant peak responses fall within the scatter of the responses in Standard trials, as for the neuron in Figure 8. Similar observations pertained to the spiking responses as well (data not shown).

We tested whether large responses to Standards tended to occur immediately following Deviants. In Figure $9 A$, these trials are marked in dark blue. It is clear from the scatter plots that these trials also have wide distribution and do not ne- 
A1

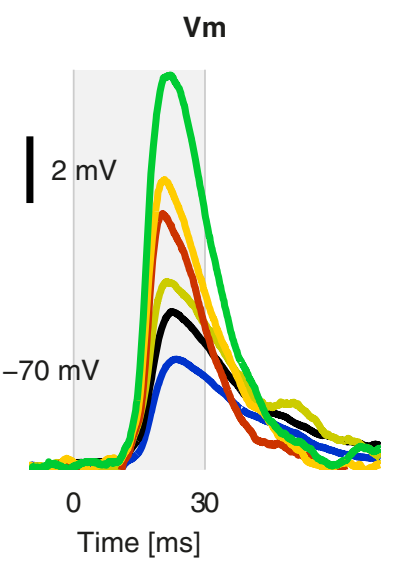

B1
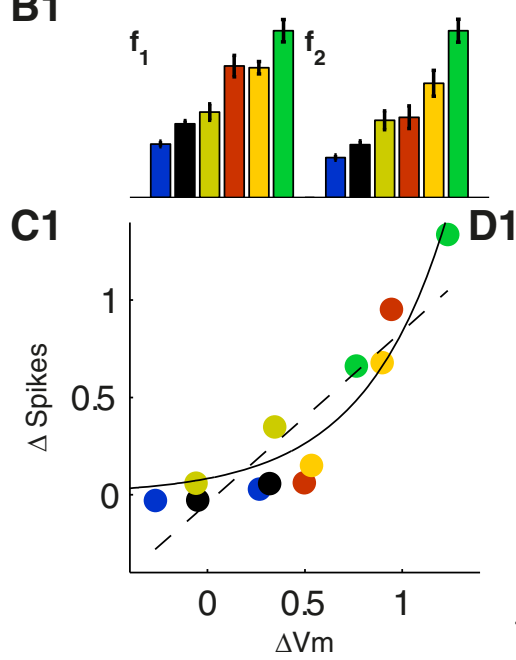

1.

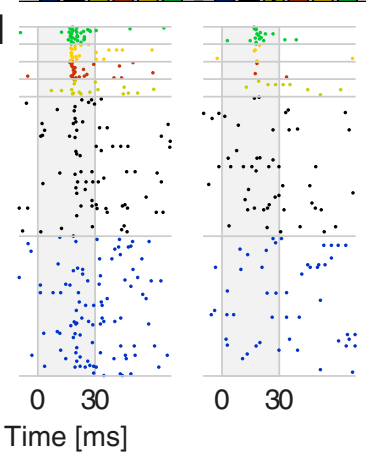

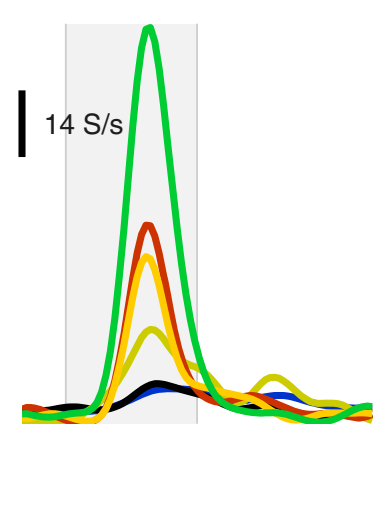

A2

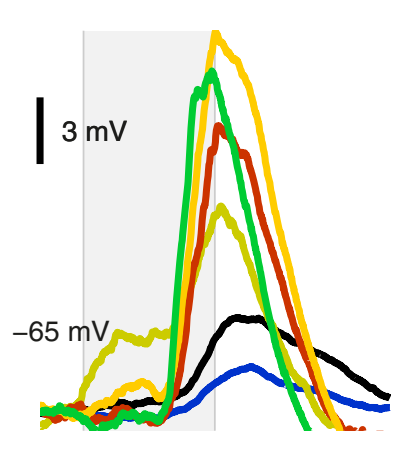

Spikes

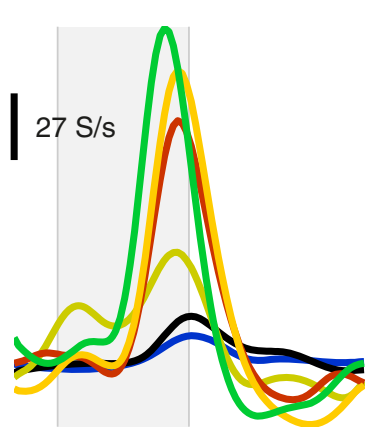

B2

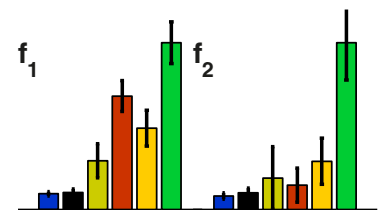

C2
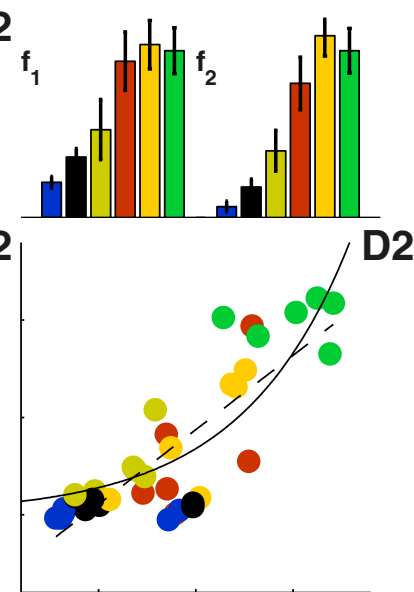

D2
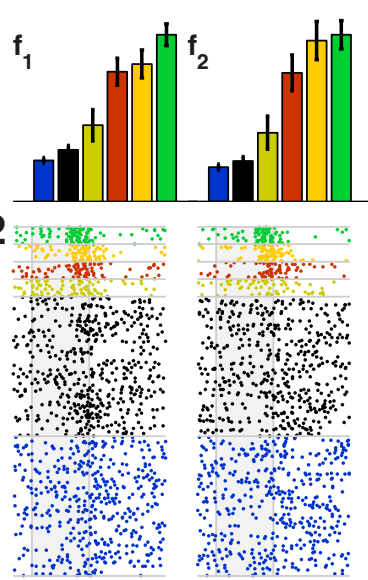

$0 \quad 30$

Time $[\mathrm{m \$}$

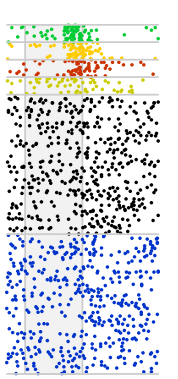

$0 \quad 30$

Figure 7. Comparing SSA in membrane potential and spiking responses of two typical neurons. A1-D1, The first neuron is marked in Figure $6 \mathrm{~A}$ with red circle above the main diagonal $(\Delta f=$ 44\%). A2-D2, The second neuron is marked in Figure $6 A$ with a red circle below the main diagonal. A1, A2, Average membrane potential responses (left) and smoothed PSTHs for the spiking responses (right) to $f_{1}$ and $f_{2}$ in all six sequences. B1, B2, Peak responses for membrane potential (left) and spiking (right) responses. C1, C2, Comparing membrane potential (abscissa) and spiking (ordinate) responses to all sequences available for each neuron ( $C 1,12$ responses, two frequencies with $\Delta f=44 \%$ in six sequences; $C_{2}, 36$ responses for two frequencies, from $3 \Delta f$ values in 6 sequences) with a linear (dashed line) and exponential (full line) fits. D1, D2, Raster plots of the spiking responses $(\Delta f=44 \%)$.

cessarily correspond to the largest responses to Standard presentations.

We quantified these observations using the responses of all neurons that did not spike ( $n=17)$, since we did not want to include data modified by spike clipping; spike clipping imposed an upper bound on the peak response values, nominally at spike threshold, and therefore the sensory reversal potential could not be identified in such neurons. Figure $9 B$ shows the average response for Standards as a function of their sequential position after a Deviant. The averages were calculated for all neurons, and the response was quantified by the difference between the average peak response and the average baseline value for all trials of that neuron. The average response to the Deviant is plotted in red, and that of the first Standard following a Deviant is plotted in dark blue. The average response to Standards at other sequential positions is plotted in light blue. The responses to the first Standard after a Deviant were indeed slightly larger than to the next few Standards. This one-trial effect is consistent with previous results. A three-way ANOVA on the sequential positions of the Standards after Deviants, with recording site and trials as random factors, was significant $\left(F_{(7,6324)}, p=0.027\right)$.

The scatter plots in Figure $9 A$ also suggested another sequential effect: the baselines just preceding the first Standard after a
Deviant tended to be higher in comparison with the overall distribution, indicating a small increase in the baseline values after a Deviant. We examined this increase in Figure $9 C$, plotting the average baseline levels preceding each tone as a function of its sequential position. As above, to combine the responses across neurons, we subtracted from each neuron its own average baseline, calculated over all trials. Remarkably, the baseline value of the first trial following a Deviant was indeed higher than the baseline value just preceding the next few trials. This increase in the baseline is sufficient to account for the increase in the average response to the first Standard following a Deviant (Fig. 9B, dark blue). Such sequential effects have not been reported previously in single-neuron recordings, to the best of our knowledge. Although significant, both sequential effects are rather small.

\section{Adaptation of excitation in narrow frequency bands}

We demonstrated that Deviant responses were smaller on average than Deviant-alone responses. This observation suggests that presentations of the Standard tones adapted the Deviant responses even when the two tones in the sequence were separated by half an octave ( $\Delta f=44 \%)$. Taaseh et al. (2011) made similar observations with respect to local field potentials (LFPs) and multiunit responses recorded in the same preparation. They de- 

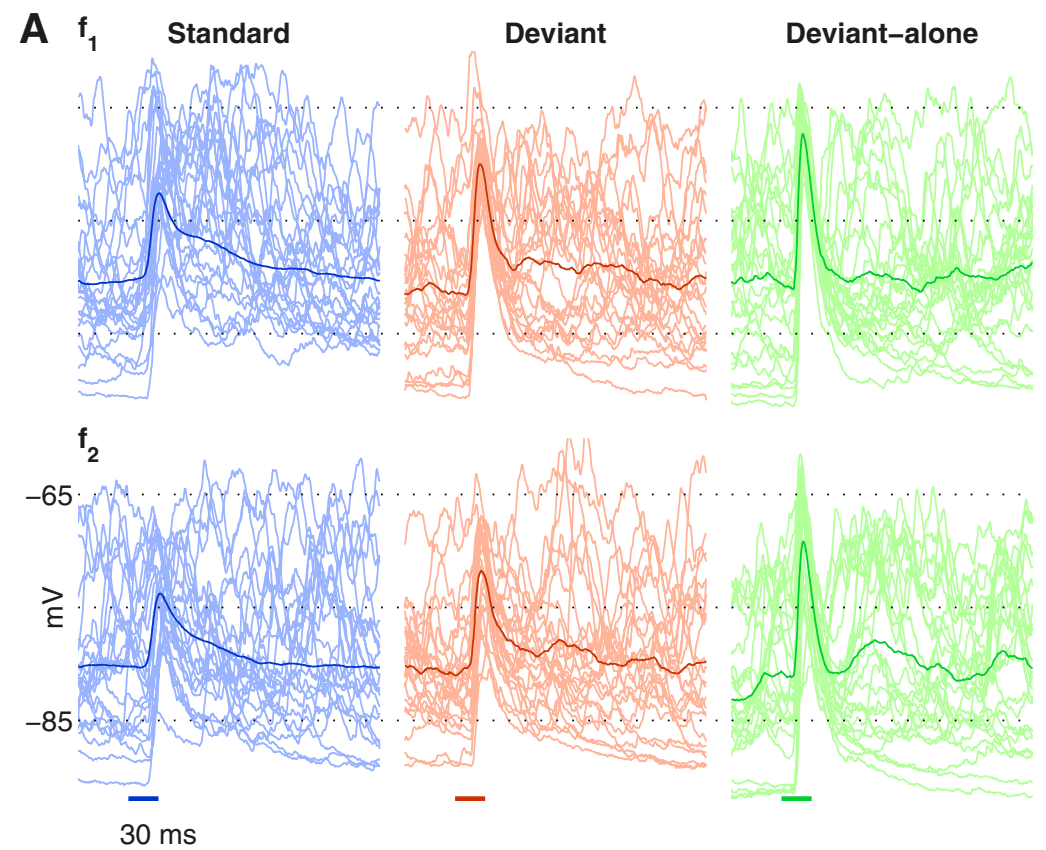

B
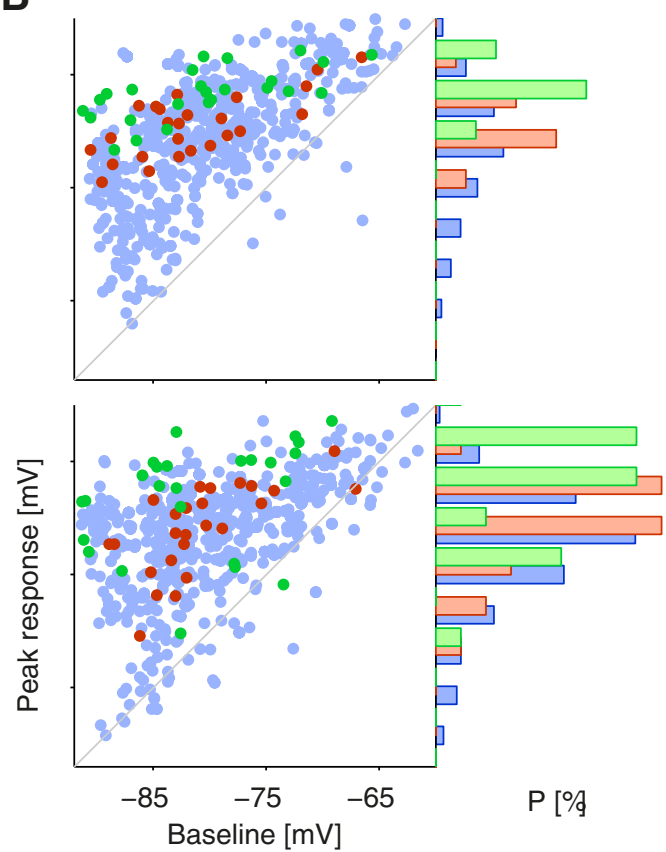

Figure 8. Single-trial analysis in one neuron (same neuron as in Fig. 1). A, Average responses (dark color traces) and single trials (light color traces) in Standard, Deviant, and Deviant-alone conditions for $f_{1}$ (top) and $f_{2}$ (bottom). All 25 trials of the Deviant and Deviant-alone conditions, and 25 randomly selected trials of the Standard condition are displayed. Stimulus duration ( $30 \mathrm{~ms}$ ) is marked as a horizontal colored line at the bottom. $\boldsymbol{B}$, Membrane potential values at the peak response after tone onset in all trials (ordinate) plotted against baseline membrane potential (abscissa) for all trials of the three sequences in $\boldsymbol{A}$ ( $y$-axis is the same as in $\boldsymbol{A}$ ). Histograms show distribution of the peak responses.

veloped a model for the responses that assumed adaptation of excitation in narrow frequency channels. Here, we fitted the same model to the membrane potential and spiking responses of each neuron separately. The data used for fitting the model included all conditions tested at all $\Delta f$ values, up to 48 data points for each neuron. We included all sets that had significant responses in the Deviant-alone condition.

Figure 10 plots the predicted responses against the observed responses, normalized by the responses to the Deviant-alone condition, for all neurons. Generally, the model approximated the data for both membrane potentials (Fig. 10A) and spikes (Fig. $10 B)$. The predicted responses approximated well the observed responses and kept the relative order of the responses in the different conditions (i.e., Standard, Equal, Diverse-narrow, Deviant, Diverse-broad, and Deviant-alone).

To quantitatively measure the goodness of fit of the model to the data, we used the ratio of $\varepsilon^{2}$ and the sum of the squared SEs $\left(s e^{2}\right.$; for fit ratios, see Taaseh et al. (2011) and Materials and Methods). The histogram of the fit ratios for all recording sites is shown in Figure $11 \mathrm{~A}$ (separately for membrane potentials and spikes). If the model were correct, fit ratios would follow the $F(n, n)$ distribution, where $n$ is the number of responses that were fitted by the model in each site. Since $n$ varied from site to site, Figure $11 A$ includes a weighted $F$ distribution, where each $F(n, n)$ distribution was weighted by the probability of observing that $n$ in the data. Among all neurons, 34 of $47(72 \%)$ had the fit ratio for the membrane potential data and 17 of $20(85 \%)$ had the fit ratio for the spike data within the $95 \%$ point of the expected distribution. Figure $11 B$ plots the fit ratios calculated for membrane potentials (abscissa) and spikes (ordinate). We found no significant difference between these fit ratios ( $p=$ 0.14 , paired $t$ test).
The main parameter of interest was the half-width of the adaptation band ( $\sigma$; see Materials and Methods). Its distribution across neurons is shown in Figure 11C. For membrane potential responses, the median of $\sigma$ was 0.19 octave, with an interquartile range of $0.12-0.32$ octave. These estimates correspond well with the values estimated by Taaseh et al. (2011) for LFPs, and are close to the critical band estimates in rats (approximately one-third of an octave; Malmierca et al., 2008). Thus, the adaptation bands summed by the cortical neurons may correspond to critical band inputs from subcortical stations. For the spiking responses, the estimated bandwidths were substantially larger: the median was 0.45 octave, with an interquartile range of $0.23-0.89$ octave. Figure $11 D$ compares the width of the adaptation channels derived from the membrane potentials (abscissa) against the values derived from the spiking responses (ordinate) for all neurons that had significant spiking responses to tones $(n=27)$. The difference between the estimated half-width of the adaptation bands for spikes and for membrane potential responses was significant (paired $t$ test: $t=5.3, \mathrm{df}=19, p=0.00004$ ). To verify that the apparent increase in the width of the adaptation channels was not due to the effects of spontaneous firing rates in the spiking responses (Fig. 6C), we fitted the model again to the spiking responses after correcting the responses by the baseline firing rates and found similar results (data not shown). For consistency with Taaseh et al. (2011), Figures 10 and 11 display the model results for the spiking responses without baseline correction.

Although it captured a significant amount of the variability of the responses, the model showed some consistent discrepancies with the data. The most important failure of the model involved the relationships between the responses in the Deviant and in the Diverse-broad conditions. This is illustrated in Figure 12A, which compares the membrane potential responses (shown here for 
A
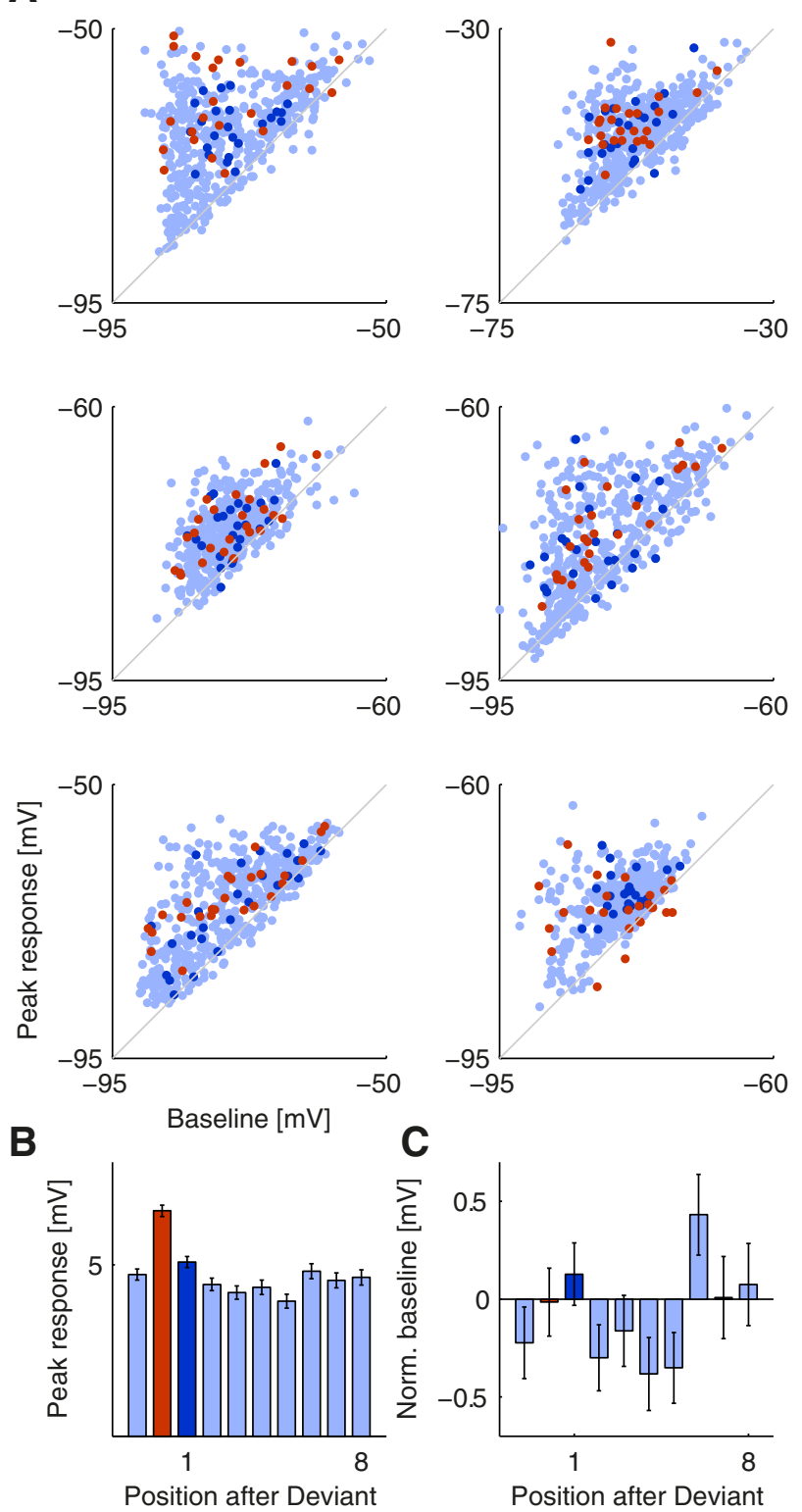

Figure 9. $\quad \boldsymbol{A}$, Membrane potential values at the peak response after tone onset in all trials (ordinate) plotted against baseline membrane potential (abscissa) for all trials from six neurons that did not spike. Only $f_{1}$ responses are shown. Dark blue, First Standards following a Deviant; light blue, all other Standards; red, Deviants. $\boldsymbol{B}$, Average peak responses to the last Standard before Deviant (light blue), Deviant (red), and the first eight Standards following a Deviant as a function of their sequential positions. The response to the first Standard after a deviant is marked in dark blue. Data are from all neurons that did not spike $(n=17)$. Responses were normalized by subtracting the average baseline for each neuron. Error bars are SEs. $\boldsymbol{C}$, Same as $\boldsymbol{B}$, for the average normalized baseline membrane potential at stimulus onset. Baseline was normalized by subtraction of the average baseline, across all trials, for each neuron.

$\Delta f=44 \%)$ to these two conditions. Generally, the observed responses in the Deviant and Diverse-broad conditions were comparable to each other (Fig. 12A, left), with approximately half the neurons (35 of 67) showing larger responses to the Deviant than to the Diverse-broad condition. In contrast, the model predicted the responses in the Diverse-broad condition to be consistently larger than in the Deviant condition (Fig. 12A, right). This bias in the prediction was highly consistent: 0 of 61 models predicted Deviant $>$ Diverse-broad responses. This result is important, since Deviant $>$ Diverse-broad is a common test for true deviance detection (Jacobsen et al., 2003). The data in Figure 12A shows that a model of adaptation in narrow frequency channels consistently fails this test, whereas many neurons pass it.

Comparing the responses in the Deviant and in the Diversebroad conditions between the observations and the model, it seems that the predicted responses to the Deviants were too small on average, while those in the Diverse-broad condition were too large (Fig. 10: Deviant responses, in red, are often below the diagonals, while Diverse-broad responses, in yellow, tend to be above the diagonals). Thus, the inclusion of the Deviant responses as part of the training data to the model presumably pulled the predictions to the Deviant responses up, although the model could not quite correctly predict their size. Conversely, including the Diverse-broad responses in the training data to the model presumably pulled these predictions down, although the model often ended up overpredicting them. We tested these ideas by retraining the models while removing the responses in either the Deviant or the Diverse-broad conditions. Figure 12B displays the differences between the predictions from the full model and from the model with the appropriate condition left out of the training data as box plots. Excluding the Deviant responses from the training set resulted in a decrease of the predicted Deviant responses (Fig. 12B, red: the distribution of differences is mostly $>0$ for both spikes and membrane potential), suggesting that, indeed, including the Deviant responses in the training data pulled up these estimates. In other words, quite consistently, the observed Deviant responses were larger than those predicted by the model trained on all other conditions. The Diverse-broad responses showed the opposite effect: excluding the responses in the Diverse-broad condition from the training set resulted in a slight increase in the predicted Diverse-broad responses (Fig. $12 B$, yellow: the distribution of differences is mostly $<0$ ). Thus, the observed Diverse-broad responses were somewhat smaller than the model predicted when given the responses in all other conditions, although this effect was weaker.

\section{Discussion}

Here we studied SSA at the level of the membrane potential and spiking responses of neurons in rat auditory cortex. Remarkably, SSA at the level of the membrane potential was present for frequency differences as small as $4 \%$ and when the interval between stimuli was as long as $1200 \mathrm{~ms}$. The transformation from membrane potential to spiking activity increased the contrast between Standards and Deviants, due to an iceberg effect caused by the spiking threshold. Finally, we reproduced the results of Taaseh et al. (2011), showing that a model of adaptation in narrow frequency channels accounts for cortical SSA only partially: while this model explains the larger responses to Deviants than to Standards, it fails to predict the relative size of the responses to other conditions that control for tone rarity. In particular, the model consistently under-predicted the responses to Deviants (given the responses to all other conditions). The finding that Deviant responses are consistently larger than predicted by this model raises the possibility that they are boosted by other mechanisms, suggesting the presence of true sensitivity to stimulus deviance in auditory cortex.

These results are generally consistent with previous studies of SSA in auditory cortex using a number of neural signals in a wide range of species, including well separated units in Cat A1 (Ulanovsky et al., 2003), and LFP and multiunit activity in A1s of anesthetized (Taaseh et al., 2011) and awake (von der Behrens et al., 2009) rats. These studies have demonstrated habituation to 
A
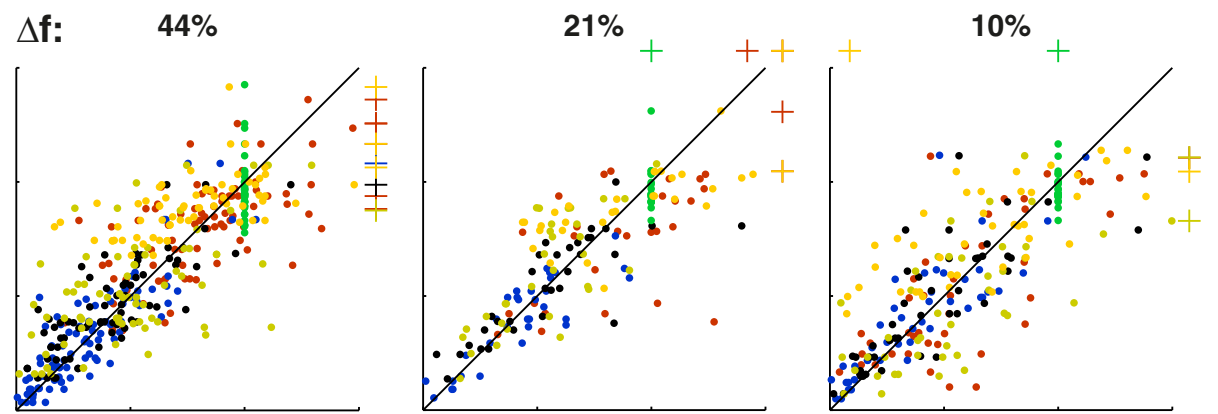

B
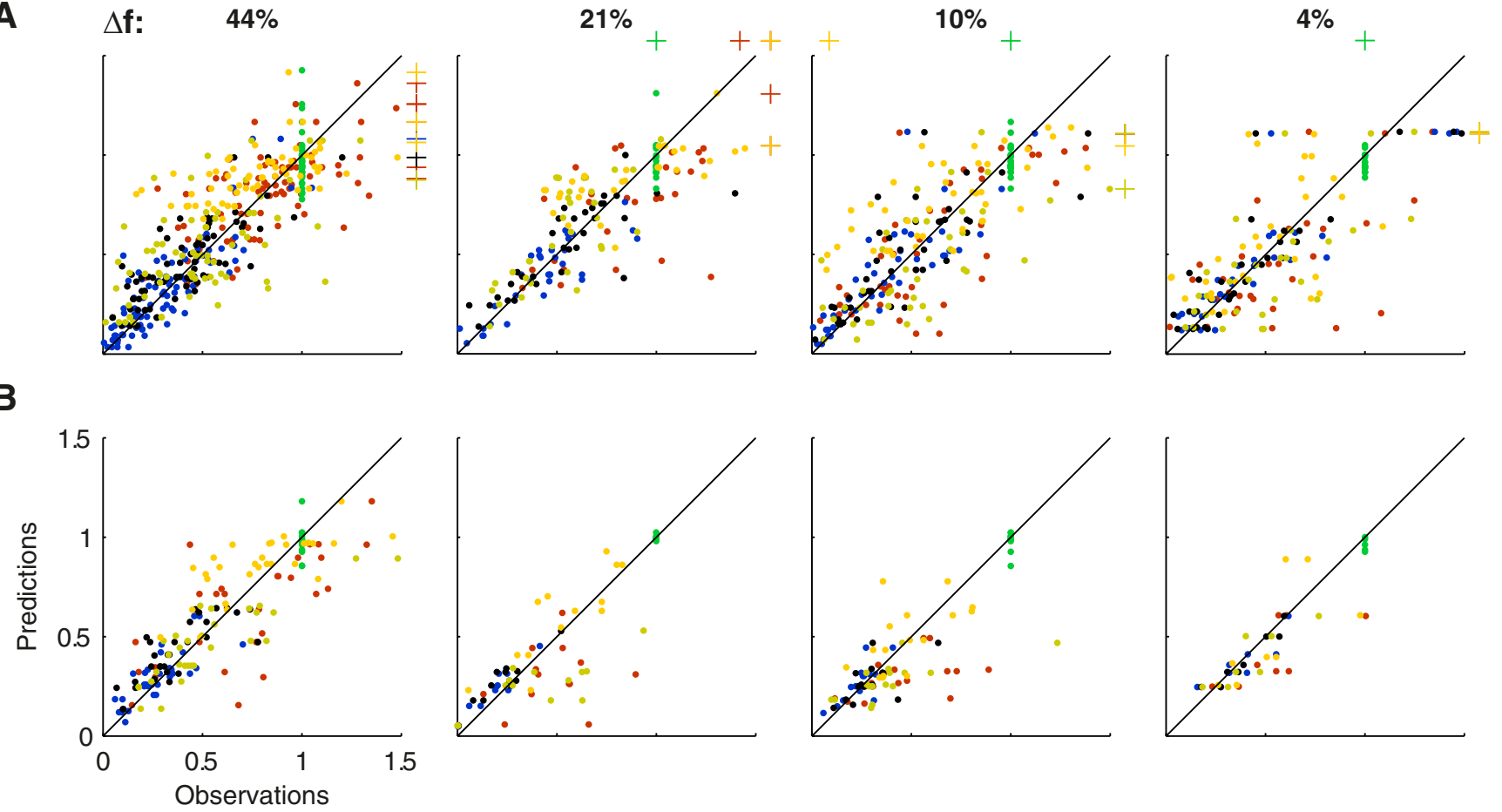

Standard

Equal

Diverse-narrow

Deviant

Diverse-broad

Figure 10. Model responses (ordinate) plotted against the measured responses (abscissa) for all $\Delta f$ conditions. A, Membrane potential responses. $\boldsymbol{B}$, Spiking responses.

A

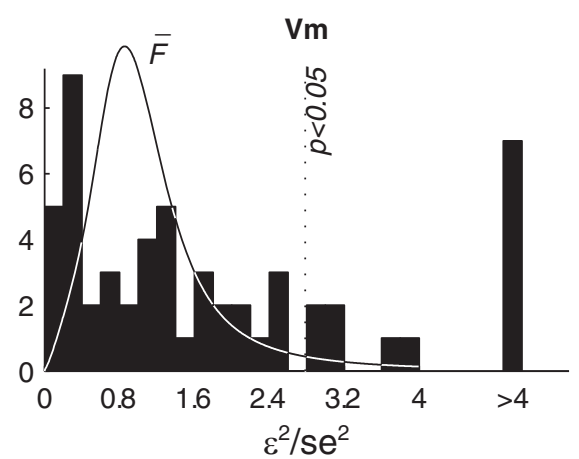

C

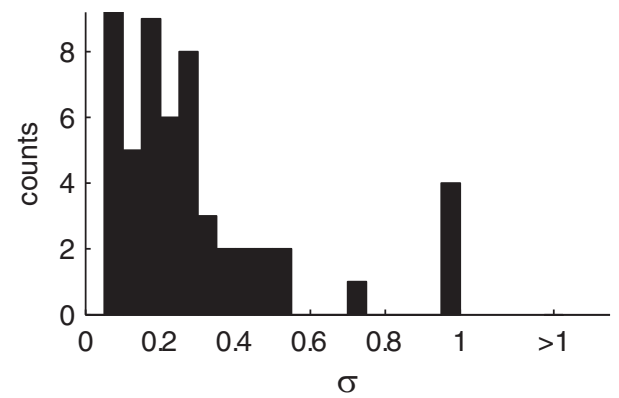

Spikes
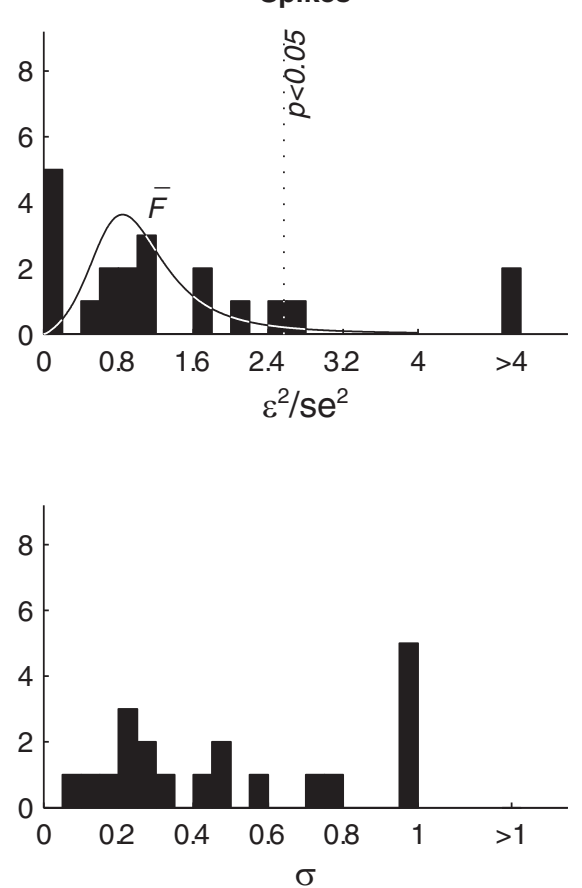

B

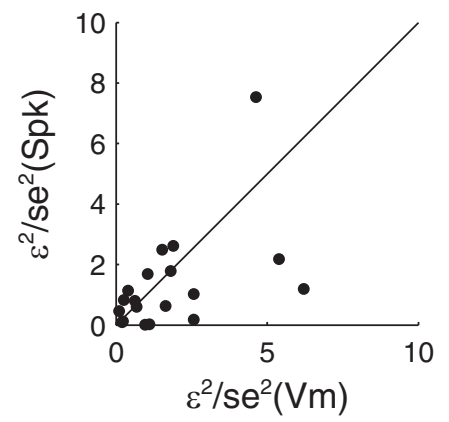

D

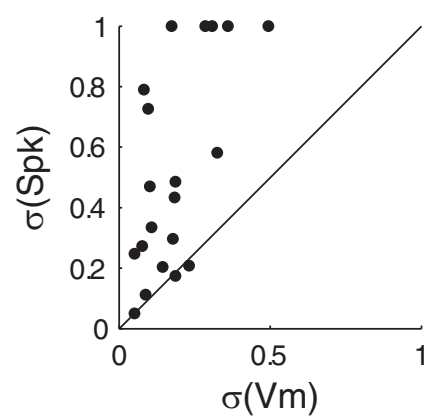

Figure 11. A, Distributions of the ratio of the $\varepsilon^{2}$ and the sum of the $s^{2}$, shown separately for $V_{m}$ and spikes. The weighted $F(n, n)$ distribution is plotted in continuous lines. The vertical dotted line indicates the $95 \%$ critical point of the expected distribution. $\boldsymbol{B}$, Scatter plot of membrane potential fit ratios (abscissa) against spike fit ratios (ordinate). C, Distributions of the half-width of the adaptation channel $(\sigma)$ shown separately for $V_{\mathrm{m}}$ and spikes. $\boldsymbol{D}$, Comparison of $\sigma$ estimated from membrane potential responses (abscissa) and spiking responses (ordinate).

the Standard that did not generalize fully to the Deviant. Here we report significant SSA for frequency separations $(\Delta f)$ as small as $4 \%$, close to the behavioral tone discrimination threshold of rats (Talwar and Gerstein, 1998; Sloan et al., 2009).
SSA and the summed inputs to cortical neurons

The average response to a Standard tone was typically smaller than the response to the same tone when Deviant, revealing a clear SSA at the level of the membrane potential (Figs. 1, 2, 3, 
A
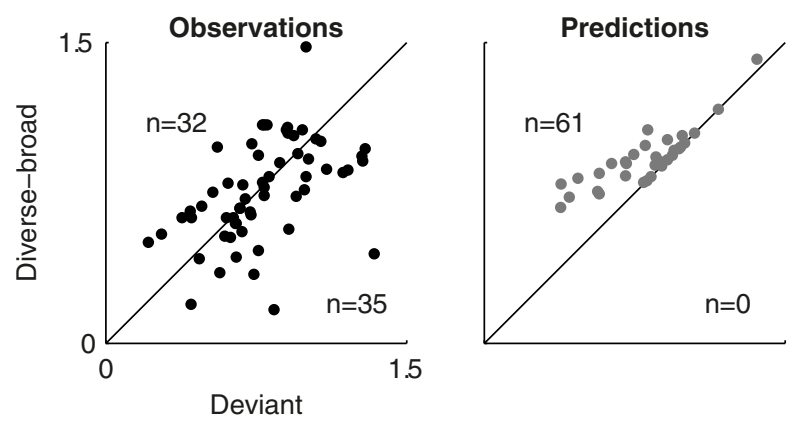

B

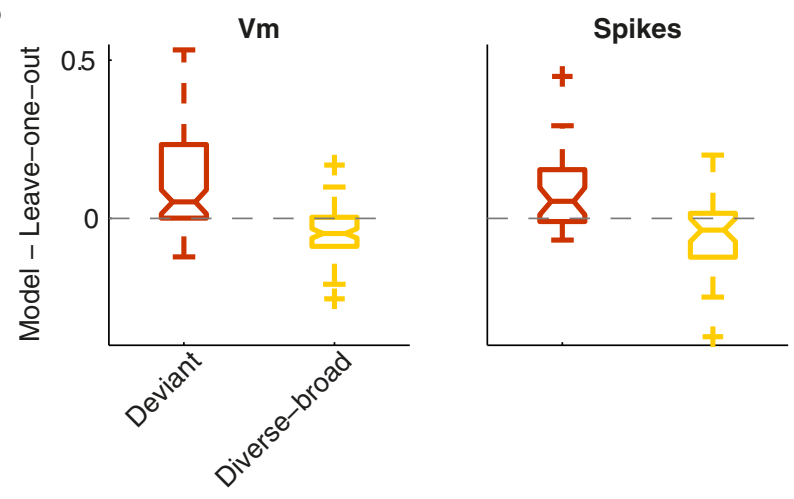

Figure 12. Comparison between Deviant and Diverse-broad responses in real neurons and in the modeled responses. $\boldsymbol{A}$, Deviant responses plotted against Diverse-broad responses for individual neurons ( $\Delta f=44 \%$ ). Left, Observed responses; right, modeled responses. $\boldsymbol{B}$, Red, Comparing responses to Deviants as predicted from the full model and as predicted from the model with the Deviant responses left out of the training set. The box plots display the distributions of the differences between the two, the large majority of which are positive. Thus, Deviant predictions generally based on all other data are too small. Yellow, Same for the Diverse-broad responses. Here the differences are mostly negative, showing that the predictions of the responses to the Diverse-broad condition, based on all other responses, are generally too large. Left, For membrane potential data $\left(V_{\mathrm{m}}\right)$; right, for spike data; both for $\Delta f=44 \%$.

5). CSI values could be quite large for $\Delta f=44 \%$ and, while smaller on average, were rarely negative even for $\Delta f=10 \%$ and $4 \%$.

These average results mask to some extent the large variability of the single-trial responses. Cortical neurons show spontaneous fluctuations in their membrane potentials. These may take the form of up and down states or of "bumpy" traces with large, short excitatory excursions, which have been described in auditory cortex of anesthetized rats and cats (Deweese and Zador, 2004; Las et al., 2005). All neurons described here had large bumps in their membrane potential traces, and in some cases they had clearly separated up and down states. The sensory stimulus could therefore occur when the membrane potential takes different values, potentially affecting sensory integration.

Indeed, stimuli gave rise to sensory responses that showed substantial size variation. Scatter plots of the sensory responses as a function of the membrane potential preceding stimulus onset (prestimulus potential) had a wedge shape. The responses were bounded from below by the prestimulus potential. They were, however, also bounded from above, essentially independently of the prestimulus potential. We interpret this upper bound in these nonspiking neurons as the reversal potential of the sensory input (Crochet et al., 2011; Sachidhanandam et al., 2013). Thus, the allowed size of the sensory responses covered the range between the prestimulus potential and the presumed sensory reversal po- tential. The rather negative effective reversal potential in Figures 7 and 8 suggests a significant contribution of inhibition in shaping the sensory input to these neurons (Wehr and Zador, 2003; Las et al., 2005; Zhou et al., 2012).

Remarkably, the responses to Standard tones covered the whole allowed range, including the largest values. Deviants, on the other hand, tended to sample the upper range of the allowed responses (Figs. 7, 8), resulting in larger average responses. Thus, the habituation to the Standard was probabilistic: within the same sequence, some Standards could evoke large responses, while others evoked small ones, even when matching the prestimulus membrane potential. Two types of sequential effects contributed to these fluctuations: the response to a Standard following a Deviant was significantly larger than to the Standard just preceding the Deviant; and the baseline preceding the first Standard after a Deviant was significantly larger than the average baseline of the next few Standards. While the first effect has been extensively documented before, the second is novel. However, both effects are quite small and could be demonstrated only by averaging across the whole neuronal population reported here. Thus, neither explains the variability between single trials.

Previous studies of the relations between membrane potential and firing rates showed that spike thresholds tend to sharpen tuning curves at the level of the membrane potential due to an iceberg effect (visual cortex: Carandini and Ferster, 2000; Carandini, 2007; Finn et al., 2007; Atallah et al., 2012; auditory cortex: Wehr and Zador, 2003). Correspondingly, we demonstrated an increase in the contrast between the responses to Standards and Deviants in the spiking responses (Fig. 6). In contrast with previous reported iceberg effects, here the increased contrast refers to stimulus context rather than to the tuning to a stimulus parameter. We speculate that inhibition may govern the size of SSA in auditory cortex (Pérez-González and Malmierca, 2012). Thus, for example, these results suggest that reducing the activity of parvalbumin-expressing inhibitory interneurons (Atallah et al., 2012) should reduce the contrast between the responses to Standards and Deviants.

\section{Mechanisms of SSA in auditory cortex}

Cross-frequency adaptation played an important role in shaping the responses to tone sequences. Thus, Deviant responses were smaller than the response in the Deviant-alone conditions, where the Standard is absent (Figs. 1, 2, 3, 4, 5, 7, 8, 9). Furthermore, Deviant responses decreased with decreasing $\Delta f$ values (Figs. 2, 3, 4).

These observations led us to study the role of adaptation in narrow frequency channels on cortical SSA. This model may represent simple fatigue of the thalamic inputs from the vMGB (Antunes et al., 2010) and/or depression of the thalamocortical synapses (Lee and Sherman, 2010). The dependence of SSA on $\Delta f$ values suggests that the width of the individual input channels is on the order of one-third to one-half octave, as expected from critical band inputs from subcortical structures.

The model makes two predictions that were directly tested here. First, the Deviant-alone responses should be larger than to any other tone sequence. Second, more subtly but quite generically, a tone in the Deviant condition should elicit smaller responses than the same tone in the Diverse-broad block (Fig. 12A). The reason is that most tone presentations in the Diverse-broad block are farther away from the test frequency than tone presentations in the Deviant block, leading to lesser cross-frequency adaptation in the Diverse-broad sequences (for a detailed analysis, see Taaseh et al., 2011). 
The model provided a reasonable fit, but had some consistent biases. In particular, the responses to the same tone in the Deviant and in the Diverse-broad conditions were comparable to each other, falsifying the very consistent prediction of the model (Fig. $12 A)$. This discrepancy could be demonstrated in other ways as well. For example, as in Taaseh et al. (2011), given the responses in all other conditions, the model predicted Deviant responses that were too small (Fig. 12B).

Thus, there may be additional mechanisms, beyond adaptation in narrow frequency channels, which contribute to SSA in auditory cortex. One possible mechanism is a dynamic adaptation channel, which changes its width according to stimulation history (Taaseh et al., 2011). In such a model, Standards may not only adapt their own responses, but also narrow the adaptation bandwidth centered on the Deviant frequency. Such a mechanism would result in less adaptation of the Deviant in an Oddball sequence, potentially reproducing our finding that the responses in the Deviant and in the Diverse-broad conditions are mostly equivalent.

\section{Implications}

SSA in auditory cortex reflects a number of successive processing mechanisms. Since vMGB neurons show little SSA, the relatively large contrast between the responses to Standards and Deviants at the level of the membrane potential of cortical neurons is presumably generated in A1. One plausible mechanism for that could be adaptation in narrow frequency channels, which reproduces the larger responses to Standards than to Deviants. However, our modeling results suggest that this is insufficient. Indeed, the finding that responses in the Diverse-broad conditions were equivalent to those in the Deviant condition, and were actually smaller than the Deviant responses in approximately half of the neurons, suggests the existence of a deviance-sensitive mechanism already at the level of the membrane potential of neurons in auditory cortex, operating in addition to adaptation in narrow frequency channels (Taaseh et al. (2011)). Finally, we demonstrated that spiking threshold significantly affected SSA, increasing the contrast between Standard and Deviant tones by virtue of an iceberg effect, which may be controlled by the level of local inhibition. We conclude that cortical SSA includes contributions of the local input to the neurons, as well as contributions of local processing and cellular mechanisms that further shape its properties.

\section{References}

Anderson LA, Christianson GB, Linden JF (2009) Stimulus-specific adaptation occurs in the auditory thalamus. J Neurosci 29:7359-7363. CrossRef Medline

Antunes FM, Malmierca MS (2011) Effect of auditory cortex deactivation on stimulus-specific adaptation in the medial geniculate body. J Neurosci 31:17306-17316. CrossRef Medline

Antunes FM, Nelken I, Covey E, Malmierca MS (2010) Stimulus-specific adaptation in the auditory thalamus of the anesthetized rat. PLoS One 5:e14071. CrossRef Medline

Atallah BV, Bruns W, Carandini M, Scanziani M (2012) Parvalbuminexpressing interneurons linearly transform cortical responses to visual stimuli. Neuron 73:159-170. CrossRef Medline

Ayala YA, Pérez-González D, Duque D, Nelken I, Malmierca MS (2012) Frequency discrimination and stimulus deviance in the inferior colliculus and cochlear nucleus. Front Neural Circuits 6:119. CrossRef Medline

Bäuerle P, von der Behrens W, Kössl M, Gaese BH (2011) Stimulus-specific adaptation in the gerbil primary auditory thalamus is the result of a fast frequency-specific habituation and is regulated by the corticofugal system. J Neurosci 31:9708-9722. CrossRef Medline

Carandini M (2007) Melting the iceberg: contrast invariance in visual cortex. Neuron 54:11-13. CrossRef Medline
Carandini M, Ferster D (2000) Membrane potential and firing rate in cat primary visual cortex. J Neurosci 20:470-484. Medline

Crochet S, Poulet JF, Kremer Y, Petersen CC (2011) Synaptic mechanisms underlying sparse coding of active touch. Neuron 69:1160-1175. CrossRef Medline

Deweese MR, Zador AM (2004) Shared and private variability in the auditory cortex. J Neurophysiol 92:1840-1855. CrossRef Medline

Farley BJ, Quirk MC, Doherty JJ, Christian EP (2010) Stimulus-specific adaptation in auditory cortex is an NMDA-independent process distinct from the sensory novelty encoded by the mismatch negativity. J Neurosci 30:16475-16484. CrossRef Medline

Finn IM, Priebe NJ, Ferster D (2007) The emergence of contrast-invariant orientation tuning in simple cells of cat visual cortex. Neuron 54:137-152. CrossRef Medline

Fishman YI, Steinschneider M (2012) Searching for the mismatch negativity in primary auditory cortex of the awake monkey: deviance detection or stimulus specific adaptation? J Neurosci 32:15747-15758. CrossRef Medline

Grimm S, Escera C (2012) Auditory deviance detection revisited: evidence for a hierarchical novelty system. Int J Psychophysiol 85:88-92. CrossRef Medline

Gutfreund Y (2012) Stimulus-specific adaptation, habituation and change detection in the gaze control system. Biol Cybern 106:657-668. CrossRef Medline

Jacobsen T, Schröger E, Horenkamp T, Winkler I (2003) Mismatch negativity to pitch change: varied stimulus proportions in controlling effects of neural refractoriness on human auditory event-related brain potentials. Neurosci Lett 344:79-82. CrossRef Medline

Las L, Stern EA, Nelken I (2005) Representation of tone in fluctuating maskers in the ascending auditory system. J Neurosci 25:1503-1513. CrossRef Medline

Lee CC, Sherman SM (2010) Topography and physiology of ascending streams in the auditory tectothalamic pathway. Proc Natl Acad Sci U S A 107:372-377. CrossRef Medline

Malmierca MS, Izquierdo MA, Cristaudo S, Hernández O, Pérez-González D, Covey E, Oliver DL (2008) A discontinuous tonotopic organization in the inferior colliculus of the rat. J Neurosci 28:4767-4776. CrossRef Medline

Malmierca MS, Cristaudo S, Pérez-González D, Covey E (2009) Stimulusspecific adaptation in the inferior colliculus of the anesthetized rat. J Neurosci 29:5483-5493. CrossRef Medline

Margrie TW, Brecht M, Sakmann B (2002) In vivo, low-resistance, wholecell recordings from neurons in the anaesthetized and awake mammalian brain. Pflugers Arch 444:491-498. CrossRef Medline

Mill R, Coath M, Wennekers T, Denham SL (2011) A neurocomputational model of stimulus-specific adaptation to oddball and Markov sequences. PLoS Comput Biol 7:e1002117. CrossRef Medline

Moshitch D, Las L, Ulanovsky N, Bar-Yosef O, Nelken I (2006) Responses of neurons in primary auditory cortex (A1) to pure tones in the halothaneanesthetized cat. J Neurophysiol 95:3756-3769. CrossRef Medline

Movshon JA, Lennie P (1979) Pattern-selective adaptation in visual cortical neurones. Nature 278:850-852. CrossRef Medline

Naatanen R, Gaillard AW, Mantysalo S (1978) Early selective-attention effect on evoked potential reinterpreted. Acta Psychol (Amst) 42:313-329. CrossRef Medline

Netser S, Zahar Y, Gutfreund Y (2011) Stimulus-specific adaptation: can it be a neural correlate of behavioral habituation? J Neurosci 31:1781117820. CrossRef Medline

Pérez-González D, Malmierca MS (2012) Variability of the time course of stimulus-specific adaptation in the inferior colliculus. Front Neural Circuits 6:107. CrossRef Medline

Pérez-González D, Malmierca MS, Covey E (2005) Novelty detector neurons in the mammalian auditory midbrain. Eur J Neurosci 22:2879-2885. CrossRef Medline

Reches A, Netser S, Gutfreund Y (2010) Interactions between stimulusspecific adaptation and visual auditory integration in the forebrain of the barn owl. J Neurosci 30:6991-6998. CrossRef Medline

Sachidhanandam S, Sreenivasan V, Kyriakatos A, Kremer Y, Petersen CC (2013) Membrane potential correlates of sensory perception in mouse barrel cortex. Nat Neurosci 16:1671-1677. CrossRef Medline

Sloan AM, Dodd OT, Rennaker RL 2nd (2009) Frequency discrimination in 
rats measured with tone-step stimuli and discrete pure tones. Hear Res 251:60-69. CrossRef Medline

Taaseh N, Yaron A, Nelken I (2011) Stimulus-specific adaptation and deviance detection in the rat auditory cortex. PLoS One 6:e23369. CrossRef Medline

Talwar SK, Gerstein GL (1998) Auditory frequency discrimination in the white rat. Hear Res 126:135-150. CrossRef Medline

Thomas JM, Morse C, Kishline L, O'Brien-Lambert A, Simonton A, Miller KE, Covey E (2012) Stimulus-specific adaptation in specialized neurons in the inferior colliculus of the big brown bat, Eptesicus fuscus. Hear Res 291:34-40. CrossRef Medline

Ulanovsky N, Las L, Nelken I (2003) Processing of low-probability sounds by cortical neurons. Nat Neurosci 6:391-398. CrossRef Medline

Ulanovsky N, Las L, Farkas D, Nelken I (2004) Multiple time scales of adaptation in auditory cortex neurons. J Neurosci 24:10440-10453. CrossRef Medline von der Behrens W, Bäuerle P, Kössl M, Gaese BH (2009) Correlating stimulus-specific adaptation of cortical neurons and local field potentials in the awake rat. J Neurosci 29:13837-13849. CrossRef Medline

Wehr M, Zador AM (2003) Balanced inhibition underlies tuning and sharpens spike timing in auditory cortex. Nature 426:442-446. CrossRef Medline

Wehr M, Zador AM (2005) Synaptic mechanisms of forward suppression in rat auditory cortex. Neuron 47:437-445. CrossRef Medline

Yaron A, Hershenhoren I, Nelken I (2012) Sensitivity to complex statistical regularities in rat auditory cortex. Neuron 76:603-615. CrossRef Medline

Zhao L, Liu Y, Shen L, Feng L, Hong B (2011) Stimulus-specific adaptation and its dynamics in the inferior colliculus of rat. Neuroscience 181:163174. CrossRef Medline

Zhou Y, Mesik L, Sun YJ, Liang F, Xiao Z, Tao HW, Zhang LI (2012) Generation of spike latency tuning by thalamocortical circuits in auditory cortex. J Neurosci 32:9969-9980. CrossRef Medline 